\title{
Global static electrospheres of charged pulsars
}

\author{
J. Pétri ${ }^{1}$, J. Heyvaerts ${ }^{1}$, and S. Bonazzola ${ }^{2}$ \\ 1 Observatoire de Strasbourg, 11 rue de l'Université, 67000 Strasbourg, France \\ 2 DARC, Observatoire de Meudon, Place Jules Janssen, 92195 Meudon, France
}

Received 21 August 2001 / Accepted 14 December 2001

\begin{abstract}
In this paper we present self-consistent models of the magnetosphere of inactive, charged, aligned rotator pulsars. We have devised an efficient semi-analytical and numerical algorithm to construct such models. The only free parameter is the total charge of the system. These charge-separated "electrospheres" consist of an equatorial belt carrying charge of one sign, partially in differential rotation, and of two oppositely charged domes located over the poles which corotate with the neutron star. The dependence of the shape of these plasma-filled regions surrounding the star on the total charge of the system and of their differential rotation is investigated. It is shown that our solutions are stable to vacuum breakdown by electron-positron pair production in most of the light-cylinder volume, except perhaps in the case of millisecond pulsars. The small regions where vacuum breakdown occurs are shown to behave merely as an effective extension of the star's volume. We have also found that no permanent null-charged wind emanating from the polar caps can exist in a stationary state. Indeed, for a given total charge of the system determined by the net outgoing charged flux, the potential configuration becomes unfavorable to particles escaping to infinity. Finally, we have shown that the geometric and kinematic structure of the electrosphere is uniquely determined by the total charge of the system.
\end{abstract}

Key words. stars: pulsars: general - plasmas - MHD - methods: numerical

\section{Introduction}

The magnetosphere of an isolated solid star or planet rotating in a vacuum seemingly has a very simple structure, being just the external vacuum region of a spinning spherical conducting magnet. However, in the regime appropriate to fast rotating magnetized neutron stars, it is a lot more complex. In a similar laboratory setup, electric charge would be distributed in the conducting body, in particular at its surface as surface-charges that would be retained in the body by a large enough extraction potential. It has however been recognized that such surface charges can be extracted even from the very magnetized crust of neutron stars by the intense electric field which appears at its surface (Goldreich \& Julian 1969). We assume this to be equally true for electrons and ions, although the case is less clear for iron nuclei than it is for electrons (Ruderman \& Sutherland 1975). Then the external region does not remain void. The determination of the structure of fields and charged flows outside of the star still remains a partially unsolved problem.

This paper, and the following in this series, aim at defining the electric structure of isolated neutron star magnetospheres in different physical regimes. We consider

Send offprint requests to: J. Pétri,

e-mail: petri@newb6.u-strasbg.fr here the aligned rotator model of a pulsar, in which the magnetic field of the star is dipolar. Following Goldreich \& Julian (1969), the stationary state reached by this system is usually viewed as consisting of a closed magnetosphere and open magnetic field structures rooted at its polar caps. Both regions are supposedly filled with electron-positron plasma, except perhaps at very small gaps. An outwards charged relativistic plasma wind is assumed to be present on field lines rooted at the polar caps that reach the light cylinder and extend beyond it. The physics of this relativistic wind has been extensively studied (Michel 1973, 1974; Goldreich \& Julian 1970; Beskin et al. 1993) and is still the subject of active research concerning the geometry of the flow (Li et al. 1992; Bogovalov 2001) and the selfconsistent determination of the total current in the system (Contopoulos et al. 1999). Unless the star is heavily charged, the sign of the extracted charges is, in the case of a dipolar field, uniform and the same over both northern and southern polar caps. The system would then globally lose charge unless the current somehow closes back on the star. Ways of achieving this have been suggested (Mestel 1984; Hofmann et al. 1996; Beskin et al. 1993) which all rely on relativistic processes such as radiation reaction or the breakdown of the electric drift approximation. Otherwise, there would be a steady charging up of the star until net charge loss is quenched by the growing 
unipolar electric field and the star's environment finds a state of electrostatic equilibrium.

This latter view of neutron star's environment has also been studied by a number of authors but has altogether received less attention than plasma-filled magnetospheres and pulsar wind models. This is due in part to the fact that an aligned rotator pulsar in a state of electrostatic equilibrium is inactive. In wind models, the space around the neutron star is supposedly entirely filled with leptonic plasma that must be self consistently produced by a pair avalanche process. This raises the question of what happens when pair creation activity ceases or when it is not possible, and also of what aligned rotators would look like if pair creation did not exist at all.

Here we study the structure of the environment of rotating neutron stars that have reached a state in which axisymmetric rotating charged plasma has found an equilibrium between the electromotive and electric fields, partly self-generated, in which it is sitting. This we describe for brevity as an electrostatic equilibrium. We refer to the associated solution as an electrosphere. We shall rely on the electrostatic approximation, neglecting self-created magnetic fields generated by the rotating charged plasma. This is correct as long as the electrosphere remains confined to well within the light cylinder.

The limits of "activity" in the parameter space of aligned rotating neutron stars is set by the stability of these inactive structures both to vacuum sparking, which we discuss in this paper, and to global electromagnetic perturbations which we shall discuss in a forthcoming paper. The construction of stationary models is a prerequisite to this study.

In Goldreich and Julian's picture (1969), the closed magnetosphere is in electrostatic equilibrium and all its volume is filled with charged plasma. This view has been challenged, and the possibility of extended vacuum gaps has been discussed by many authors (Holloway 1973; Holloway \& Pryce 1981; Ruderman \& Sutherland 1975; Cheng et al. 1976). It has even been shown that a closed magnetosphere contained within the light cylinder and totally plasma filled is unstable to the opening of wide gaps between the positively and negatively charged regions (Thacker et al. 1998).

The effect of a net total charge of the neutron star has been considered by Jackson (1976) who has shown that domes consisting of electrically trapped charged particles are then likely to form above the star's polar regions. Jackson did not obtain a complete self consistent solution, though. Pilipp (1974) made the important point that no consistent solution exists in which the electrosphere would only consist of plasma surrounding the star and corotating with it, whatever the shape of the interface of the plasma filled region with the surrounding vacuum. Pilipp's theorem does not exclude the possibility that the electrosphere consists of a corotating part, connected to the star itself, and a region separated from it by a certain potential drop along field lines. This latter region would in general be differentially rotating.
The first successful attempt at finding a completely self consistent solution for the structure of an electrosphere was made by Krause-Polstorff \& Michel (1985a, 1985b), who numerically conducted an experiment by which a number of annular shaped charges are transfered to the magnetosphere and naturally find an equilibrium position. Due to the use of a limited number of charges their results were somewhat noisy. In this paper we elaborate on their approach in the same spirit by building up the electrosphere numerically by a recurrence process which however does not consist in transferring a limited number of "point" charges. Other authors (Shibata 1989a, 1989b) have conducted similar numerical constructions, but did not calculate the differentially rotating part of the electrosphere, a most important aspect with which we deal here.

\section{The model}

Our study considers an aligned rotator with a dipolar magnetic field. We assume that the external fields and flows share the axisymmetry of the driving setup. For their description we either use cylindrical coordinates $(r, \varphi, z)$ or spherical coordinates $(R, \theta, \varphi)$ built on the rotation axis, the angle $\theta$ being the colatitude. Unit vectors of the corresponding local frames are denoted by $\boldsymbol{e}$ with a subscript, such as $\boldsymbol{e}_{\mathrm{R}}$ say. Physical quantities are expressed in the MKSA system of units, $\varepsilon_{0}$ being the dielectric permittivity of vacuum and $\mu_{0}$ its magnetic permeability.

An electrosphere is a charge distribution in which, wherever there is plasma, the electric field component aligned to the magnetic field vanishes, just as it does in the supposedly perfectly conducting star itself. The electrospheric plasma is regarded as cold (models of hot chargeseparated electrospheres have been calculated by Neukirch 1993). This plasma can be distributed in volumes separated by vacuum regions, which we refer to as vacuum gaps, or gaps for short. The vacuum/cold plasma interface is sharp. At equilibrium this interface should be force free in the sense that there should be no electric force acting along the direction of the magnetic field at it. In the gaps the electric potential is a solution of Laplace's equation and the electric field may have a component along the magnetic field. Another requirement is that the solution be accessible, in the sense that there exist some physically possible way of building it up by transferring the charges from the star to where they should eventually be found, starting from, say, an initial state where there is vacuum outside of the star.

\section{Assumptions and method for building a solution}

\subsection{Assumptions}

The following assumptions are used throughout the paper:

- the neutron star is a perfect spherical conductor of radius $R_{*}$, source of an external dipolar magnetic field, 
of strength $B_{*}$ at its north pole, which rotates around its magnetic moment at a rate $\Omega_{*}$ (aligned rotator);

- relativistic effects near the light cylinder as well as the magnetic field induced by the motion of the electrospheric charges are neglected;

- charged particles move according to the electric drift approximation;

- neglecting all forces other than electromagnetic, particles will eventually find equilibrium when the condition

$\boldsymbol{E}+\boldsymbol{v} \times \boldsymbol{B}=\mathbf{0}$

will be met, so that the charged fluid velocity $\boldsymbol{v}$ equals the electric drift velocity

$\boldsymbol{v}_{\mathrm{D}}=\frac{\boldsymbol{E} \times \boldsymbol{B}}{B^{2}} ;$

- the electrospheric plasma is confined well within the light cylinder, which justifies some of the above assumptions;

- any charged particle can escape freely from the stellar crust whatever its nature, which implies that the work function is negligible compared to the electric potential developed in vacuo across the surface of the solid crust.

As a result of these assumptions, the dipolar magnetic field is supposedly unaltered by electrospheric currents. Let the magnetic flux through a circle centered on the axis and passing at the point of cylindrical coordinates $r$ and $z$ be $2 \pi a(r, z)$. The value of the flux function $a$ can be used to label the magnetic surfaces, defined as being the locus of all such circles encompassing the same flux, $2 \pi a$. The magnetic field is expressed in terms of $a(r, z)$ by:

$\boldsymbol{B}(r, z)=\frac{1}{r} \nabla a(r, z) \times \boldsymbol{e}_{\varphi}$.

The parameters which describe the star-electrosphere system are $R_{*}, B_{*}, \Omega_{*}$ and the system's total charge $Q_{\text {tot }}$. However the star's radius enters only as a scale, as do also the polar magnetic field and the rotation rate. But for these trivial scaling factors, the electrospheric structure depends only, as we shall see, on the dimensionless electric charge of the system, i.e. on the ratio $Q_{\text {tot }} / Q_{\mathrm{c}}$ where $Q_{\mathrm{c}}=\frac{4 \pi}{3} \varepsilon_{0} \Omega_{*} B_{*} R_{*}^{3}$ is the central point charge of the neutron star in the presence of a dipolar magnetic field (Michel 1991). For dipolar magnetic fields, this charge arises from the corotation charge density defined in Eq. (22) below, which then has an integrable singularity at the origin, corresponding to a central point charge $Q_{\mathrm{c}}$. The solutions we are seeking for, if they exist, then form a one-parameter family. For definiteness we assume $B_{*}$ and $\Omega_{*}$ to be positive with no loss of generality, since the extraction of both positive or negative surface charge is assumed to be equally easy. Any other situation would be trivially dealt with by an appropriate change of charge sign.

\subsection{Iterative scheme for obtaining solutions}

Let us now describe our iterative scheme to converge to a solution and introduce the physical quantities which characterize the structure of an electrosphere. We assume the initial situation to be that which exists when electric charges have not escaped out of the star's crust. The corresponding external and internal electric potentials are easily obtained. Our algorithm then works along the following steps:

1. transfer a fraction of the stellar surface charge along magnetic field lines to the appropriate chargeseparated plasma region;

2. evaluate the total electric potential $\phi(a, \theta)$ due to this new charge distribution;

3 . find the corresponding differential rotation rate $\Omega(a)$ in the non-corotating part of the electrosphere;

4. deduce the associated differential Goldreich-Julian charge density $\rho_{\neq}(a, \theta)$ there;

5 . find the new volume occupied by plasma in each magnetic flux tube which has received new charge;

6. go back to step 2 until $\phi$ and $\Omega$ are self-consistently determined;

7. go back to step 1 until no stellar surface charge remains.

Bearing this in mind we now go into somewhat more detail and present the equations involved in the construction of these electrospheric models.

\subsection{Initialization of the iterative process}

We begin by considering a conducting sphere, source of a dipolar magnetic field, rotating at a rate $\Omega_{*}$ surrounded by vacuum. The dipolar magnetic field at a point of spherical coordinates $(R, \theta)$ is expressed as:

$\boldsymbol{B}_{\mathrm{ext}}=\frac{B_{*} R_{*}^{3}}{2 R^{3}}\left(2 \cos \theta \boldsymbol{e}_{\mathrm{R}}+\sin \theta \boldsymbol{e}_{\theta}\right)$.

The rotation gives rise in the interior of the star to an electromotive field expressed as:

$\boldsymbol{E}_{\mathrm{int}}=\frac{\Omega_{*} B_{*} R_{*}^{3}}{2 R^{2}}\left(\sin ^{2} \theta \boldsymbol{e}_{\mathrm{R}}-2 \sin \theta \cos \theta \boldsymbol{e}_{\theta}\right)$.

Its external quadrupolar counterpart is, for a net charge $Q$,

$$
\begin{aligned}
\boldsymbol{E}_{\mathrm{ext}}= & {\left[\frac{Q}{4 \pi \varepsilon_{0} R^{2}}-\Omega_{*} B_{*} R_{*}^{5} \frac{P_{2}(\cos \theta)}{R^{4}}\right] } \\
& \times \boldsymbol{e}_{\mathrm{R}}-\Omega_{*} B_{*} R_{*}^{5} \frac{\sin \theta \cos \theta}{R^{4}} \boldsymbol{e}_{\theta}
\end{aligned}
$$

where $P_{2}$ is the Legendre polynomial of degree $2, P_{2}(x)=$ $\left(3 x^{2}-1\right) / 2$. The discontinuity of the normal component of the electric field at the surface of the star gives the surface charge density, namely:

$\sigma_{\mathrm{S}}(\theta \mid Q)=\frac{Q}{4 \pi R_{*}^{2}}-\varepsilon_{0} \Omega_{*} B_{*} R_{*} \cos ^{2} \theta$.

The huge electric field is large enough to extract any such surface charge which, by moving along magnetic surfaces until eventually they reach an equilibrium position, i.e. a region where the magnetic and electric fields are perpendicular to each other. 


\subsection{Electric structure in the presence of external charge}

\subsubsection{The potential}

To proceed with the iteration, we must calculate at each step the most significant quantity which is the electric potential $\phi$ outside of the star. We split this quantity into two parts, the first one being the vacuum electric potential created by the charges present inside the neutron star and at its surface, noted $\phi_{\mathrm{v}}$, and the second one being the electrospheric potential, $\phi_{\mathrm{el}}$, created by the charges present in the external region. Inside the star the total potential is the corotation potential

$$
\begin{aligned}
\phi_{\mathrm{c}} & =\frac{Q_{\mathrm{tot}}-Q_{\mathrm{el}}-Q_{\mathrm{imel}}-Q_{\mathrm{c}}}{4 \pi \varepsilon_{0} R_{*}}+\frac{1}{2} \frac{\Omega_{*} B_{*} R_{*}^{3}}{R} \sin ^{2} \theta \\
& =\frac{Q_{\mathrm{tot}}-Q_{\mathrm{el}}-Q_{\mathrm{imel}}-Q_{\mathrm{c}}}{4 \pi \varepsilon_{0} R_{*}}+\Omega_{*} a
\end{aligned}
$$

where $Q_{\mathrm{el}}$ and $Q_{\text {imel }}$ are the charge of the electrosphere and of its image respectively (see the exact expression below). The boundary conditions to be imposed are that the total potential $\phi$ vanishes at infinity and equals $\phi_{\mathrm{c}}$ on the stellar surface. If $\phi_{\mathrm{v}}$ is chosen such as to satisfy these two conditions, $\phi_{\mathrm{el}}$ should be imposed such that it vanishes on the stellar surface and at infinity. By using the method of images described in Jackson (1975) we find that this potential is expressed in terms of the external volume charge density $\rho_{\mathrm{el}}$ by:

$\phi_{\mathrm{el}}(\boldsymbol{r})=\frac{1}{4 \pi \varepsilon_{0}} \iiint \rho_{\mathrm{el}}\left(\boldsymbol{r}^{\prime}\right) G\left(\boldsymbol{r} \mid \boldsymbol{r}^{\prime}\right) \mathrm{d}^{3} \boldsymbol{r}^{\prime}$

where the Green's function satisfying these boundary conditions is:

$G\left(\boldsymbol{r} \mid \boldsymbol{r}^{\prime}\right)=\frac{1}{\left\|\boldsymbol{r}-\boldsymbol{r}^{\prime}\right\|}-\frac{1}{\left\|\frac{R^{\prime}}{R_{*}} \boldsymbol{r}-\frac{R_{*}}{R^{\prime}} \boldsymbol{r}^{\prime}\right\|}$.

Let $Q_{\text {el }}=\iiint \rho_{\text {el }}(\boldsymbol{r}) \mathrm{d}^{3} \boldsymbol{r}$ be the charge of the electrosphere and $Q_{\text {imel }}=-R_{*} \iiint \frac{\rho_{\mathrm{el}}(\boldsymbol{r})}{R} \mathrm{~d}^{3} \boldsymbol{r}$ that of its image (both these integrals extend over the region external to the star). The stellar potential (which satisfies Laplace's equation) is given by:

$\phi_{\mathrm{v}}(R, \theta)=\frac{Q_{\mathrm{tot}}-Q_{\mathrm{el}}-Q_{\mathrm{imel}}}{4 \pi \varepsilon_{0} R}-\frac{\Omega_{*} B_{*} R_{*}^{5}}{3 R^{3}} P_{2}(\cos \theta)$.

Taking advantage of the axisymmetry it is possible to perform the integration in Eq. (10) with respect to the azimuth $\varphi$ analytically. Let $P\left(\boldsymbol{r} \mid \boldsymbol{r}^{\prime}\right)$ be the potential at $\boldsymbol{r}$ due to an unit-charge ring placed at $\boldsymbol{r}^{\prime}$, vanishing on the stellar surface and at infinity. $P$ is simply given by an azimuthal integration of Green's function (11)

$P\left(\boldsymbol{r} \mid \boldsymbol{r}^{\prime}\right)=\frac{1}{8 \pi^{2} \varepsilon_{0}} \int_{0}^{2 \pi} G\left(\boldsymbol{r} \mid \boldsymbol{r}^{\prime}\right) \mathrm{d} \varphi^{\prime}$ and can be expressed in terms of the complete elliptic integrals of the first kind ${ }^{1}$ as

$P\left(r, z \mid r^{\prime}, z^{\prime}\right)=\frac{1}{2 \pi^{2} \varepsilon_{0}} \frac{k K\left(k^{2}\right)-l K\left(l^{2}\right)}{\sqrt{4 r r^{\prime}}}$

where the variables $k^{2}$ and $l^{2}$ are defined in terms of $\boldsymbol{r}$ and $\boldsymbol{r}^{\prime}$ by

$$
\begin{aligned}
k^{2} & =\frac{4 r r^{\prime}}{\left(r+r^{\prime}\right)^{2}+\left(z-z^{\prime}\right)^{2}} \\
l^{2} & =\frac{4 r r^{\prime} \frac{R_{*}^{2}}{r^{\prime 2}+z^{\prime 2}}}{\left(r+r^{\prime} \frac{R_{*}^{2}}{r^{\prime 2}+z^{\prime 2}}\right)^{2}+\left(z-z^{\prime} \frac{R_{*}^{2}}{r^{\prime 2}+z^{\prime 2}}\right)^{2}} .
\end{aligned}
$$

The electrospheric potential associated to the charge density distribution $\rho_{\mathrm{el}}$ finally is

$\phi_{\mathrm{el}}(r, z)=\iint 2 \pi r^{\prime} \rho_{\mathrm{el}}\left(r^{\prime}, z^{\prime}\right) P\left(r, z \mid r^{\prime}, z^{\prime}\right) \mathrm{d} r^{\prime} \mathrm{d} z^{\prime}$.

\subsubsection{The stellar surface charge density}

The stellar surface charge density $\sigma_{\mathrm{s}}$ is again obtained from the discontinuity of the component of the electric field normal to the star's surface, $E_{\mathrm{R}}=-\partial \phi / \partial R$, which is easily expressed in terms of $\phi_{\mathrm{el}}$ by:

$\frac{\sigma_{\mathrm{s}}(\theta)}{\varepsilon_{0}}=\frac{Q_{\mathrm{tot}}-Q_{\mathrm{el}}-Q_{\mathrm{imel}}}{4 \pi \varepsilon_{0} R_{*}^{2}}-\Omega_{*} B_{*} R_{*} \cos ^{2} \theta-\left.\frac{\partial \phi_{\mathrm{el}}}{\partial R}\right|_{R_{*}^{+}}$

where the last term is the limit of the indicated partial derivative when $R$ approaches $R_{*}$ by larger values.

\subsubsection{The rotation velocity}

In the plasma-filled region the relation (1) implies that magnetic field lines are equipotentials so that the electric potential $\phi$ in these regions is a function $\phi(a)$ of the flux variable $a$. The associated electric field is

$\boldsymbol{E}=-\nabla \phi=-\frac{\mathrm{d} \phi}{\mathrm{d} a} \nabla a$.

Replacing this in (2), the rotation rate of the plasma in this region is found to be:

$\Omega(a)=\phi^{\prime}(a)$.

Here as below the derivative of any function of $a$ with respect to its argument is noted by a prime.

\subsubsection{The generalized Goldreich-Julian density}

In a closed magnetosphere corotating with the star at the angular velocity $\Omega_{*}$ the charge density is the so-called Goldreich-Julian value $\rho_{\text {GJ }}$, the value of which results from

\footnotetext{
1 Defined by $K\left(k^{2}\right)=\int_{0}^{\pi / 2} \frac{\mathrm{d} \varphi}{\sqrt{1-k^{2} \sin ^{2} \varphi}}$ where the parameter $k^{2}$ varies between 0 and 1. See for example Abramowitz \& Stegun (1965).
} 
assuming local electrostatic equilibrium with the electromotive field, so that

$\boldsymbol{E}+r \Omega_{*} \boldsymbol{e}_{\phi} \times \boldsymbol{B}=0$.

The associated charge density is:

$\rho_{\mathrm{GJ}}=\varepsilon_{0} \operatorname{div}\left(\boldsymbol{B} \times r \Omega_{*} \boldsymbol{e}_{\phi}\right)$.

Expanding this by use of vector calculus relations and Ampere's equation gives

$\rho_{\mathrm{GJ}}=-2 \varepsilon_{0} \boldsymbol{\Omega}_{*} \cdot \boldsymbol{B}+\frac{r \Omega_{*}}{c^{2}} j_{\varphi}$

where $\boldsymbol{j}$ is the electric current density. At a point electrically separated from the star, by which we mean that there is a vacuum interval on the segment of field line connecting this point to the star, the potential differs from the corotation potential. The corresponding plasma density then also differs from the Goldreich-Julian density. In the sub-relativistic regime this "differential GoldreichJulian density" can be calculated from Poisson's equation written in the form

$\rho=-\varepsilon_{0} \operatorname{div}(\Omega(a) \nabla a)$.

It is expressed as:

$\rho_{\neq}(R, \theta)=-\varepsilon_{0}\left(2 \boldsymbol{\Omega} \cdot \boldsymbol{B}+r^{2} B^{2} \Omega^{\prime}(a)\right)$.

\subsubsection{Determination of the boundaries of charged electrospheric regions}

At each step of the iterative construction of the solution we know how much charge, $q_{\mathrm{D}}(a) \mathrm{d} a$ and $q_{\mathrm{P}}(a) \mathrm{d} a$ resp., is contained in the equatorial belt region (hereafter referred to as "the disk", subscript D) and in each polar dome (subscript $\mathrm{P}$ ) in the flux tube between the magnetic surfaces $a$ and $a+\mathrm{d} a$ (see Fig. 1). The volume charge densities in these regions are $\rho_{\neq}$and $\rho_{\text {GJ }}$ respectively. These quantities are thus related by $q_{\mathrm{D}}(a) \mathrm{d} a=\iiint \rho_{\neq}(\boldsymbol{r}) \mathrm{d}^{3} \boldsymbol{r}$, where the integral is extended to the volume of the disk situated between the magnetic surfaces $a$ and $a+\mathrm{d} a$, and by $q_{\mathrm{P}}(a) \mathrm{d} a=\iiint \rho_{\mathrm{GJ}}(\boldsymbol{r}) \mathrm{d}^{3} \boldsymbol{r}$ the integration now extending over the volume of one dome, also between these same magnetic surfaces. For known rotation $\Omega(a)$ and charge to flux ratios $q_{\mathrm{D}}(a)$ and $q_{\mathrm{P}}(a)$ in the disk and dome resp., we obtain from the former relation an equation which gives the latitude $\alpha(a)$ of the edge of the equatorial belt on magnetic surface $a$ as

$$
\begin{aligned}
{\left[2 \Omega(a)+a \Omega^{\prime}(a)\right] \sin ^{3} \alpha(a) } & -\left[2 \Omega(a)-a \Omega^{\prime}(a)\right] \sin \alpha(a) \\
& +\frac{a q_{\mathrm{D}}(a)}{2 \pi^{3} \varepsilon_{0} B_{*} R_{*}^{3}}=0
\end{aligned}
$$

and from the latter an equation which gives the distance $R_{\gamma}(a)$ from the origin to the edge of the dome on magnetic surface $a$ as

$R_{\gamma}(a) \sqrt{1-\frac{2 a R_{\gamma}(a)}{B_{*} R_{*}^{3}}}=R_{*} \sqrt{1-\frac{2 a}{B_{*} R_{*}^{2}}}-\frac{q_{\mathrm{P}}(a)}{4 \pi \varepsilon_{0} \Omega_{*}} \cdot$

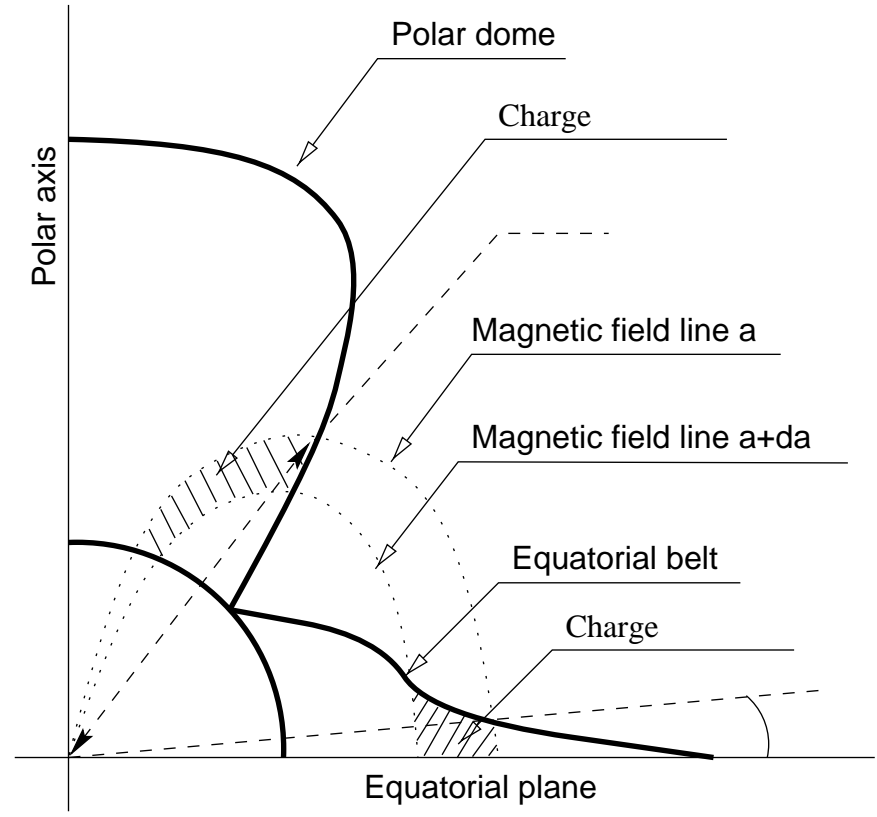

Fig. 1. Schematic representation of the geometrical shape of the electrosphere, illustrating the definition of the parameters $\alpha(a)$ and $R_{\gamma}(a)$ that describe its boundaries depending on the magnetic line $a$.

It is shown in Appendix A that in a state of full electrostatic equilibrium, as assumed for our models, no gap can exist between the stellar surface and the innermost edge of a dome, whatever the total charge $Q_{\text {tot }}$. Polar gaps of the standard pair avalanche model with wind are maintained by particle inertia. This property of electrostatic equilibria justifies that each dome is taken to be corotating and its shape uniquely determined by the radial position $R_{\gamma}(a)$ of its outer boundary on magnetic surface $a$. Its inner boundary coincides at each iteration step with the stellar surface. Figure 1 illustrates the geometry of these boundaries and the definition of the functions $\alpha(a)$ and $R_{\gamma}(a)$.

\subsubsection{Iterative charge transfer: The transfer function}

Nothing has been said about the mechanism of extraction of particles from the pulsar crust. This is because this physics is irrelevant to the mathematical iteration process by which our solutions are constructed. It is only demanded that charge extraction and transfer be indeed physically possible. The question of feasibility of charged particle emission from the surface of a neutron star is still unsolved due to the unknown value of the work function. Attempts have been made to estimate this function (Flowers et al. 1977) but yield different results (Müller 1984; Neuhauser 1987). According to Jones (1986), the cohesive energy of the atomic lattice is roughly several hundred $\mathrm{eV}$. This energy range is reached for surface temperatures $T=10^{6}-10^{7} \mathrm{~K}$, which are believed to be the temperatures of the radio-pulsar crust after cooling. Therefore we conclude that the surface temperature of the star is large enough to allow ionic (even iron nuclei) and 
electronic thermo-emission from the entire stellar crust. If however the extraction of the heavy ions were to be impossible, only the two electronically charged domes would subsist for an aligned rotator. For an anti-aligned rotator, only the equatorial disc would remain. Its structure would not be drastically altered by the absence of the dome. In what follows, we adopt Jones work function energy which implies easy charge extraction whatever their nature.

How our algorithm progressively transfers charges from the surface of the rotating neutron star to its electrosphere is described by a transfer function, $p(\theta, \lambda)$, which we made to depend on the colatitude of charge extraction $\theta$, where the surface charge density currently is $\sigma_{\mathrm{s}}(\theta)$, and on the number of the iteration step $\lambda$. It is defined as being the ratio of the surface charge, $\mathrm{d} Q_{\text {extr }}$, extracted from the pulsar's surface between colatitudes $\theta$ and $\theta+\mathrm{d} \theta$ at iteration step $\lambda$ to the surface charge $\mathrm{d} Q_{\mathrm{s}}=2 \pi R_{*}^{2} \sigma_{\mathrm{s}}(\theta) \sin \theta \mathrm{d} \theta$ present at this step between these colatitudes. So,

$\mathrm{d} Q_{\text {extr }}=p(\theta, \lambda) \mathrm{d} Q_{\mathrm{s}}$.

To ensure that the final state be indeed accessible by physically possible processes, we have imposed some restrictions on this transfer function and introduced a few specific procedures in the algorithm. For example, if some charge extracted from the surface would have, in order to reach its equilibrium position, to cross over a region filled with charges of an opposite sign, it would be physically stopped in the latter by recombination. This we take into account by diminishing the total opposite sign charge present in the flux tube where injection takes place rather than by assignating the extracted charge to a region that is not physically accessible to it.

\section{Results}

\subsection{Static electrospheres}

In order to keep all quantities close to unity in our simulations and to avoid floating-point overflow, our calculations are performed in normalized units that refer to the radius $R_{*}$, the polar magnetic field strength $B_{*}$ and to the angular velocity $\Omega_{*}$. Since we take no relativistic effect into account, the speed of light and the light cylinder radius play no explicit role and these three quantities act only as scaling factors. They do not alter anything but scales in the physical results when varied. All units derive naturally from them. In particular, the reference value for electric charge is $\varepsilon_{0} \Omega_{*} B_{*} R_{*}^{3}$. The central point charge $Q_{\mathrm{c}}$ expressed in this unit is $4 \pi / 3$.

The system has been investigated for values of the total charge which are a multiple of this central point charge of the star. The only parameter which really affects the structure of the equilibrium electrosphere is the total charge of the system $Q_{\text {tot }}$.

We computed several models with different values of this parameter but we will only discuss here a few among the most representative ones.
Figure 2 shows the main characteristics of a static electrosphere with $Q_{\text {tot }}=Q_{\mathrm{c}}$. The star is entirely surrounded by charge-separated plasma consisting of an equatorial belt, positively charged with the present convention for the sign of $B_{*} \Omega_{*}$ and of two negatively charged domes located over the polar caps. It is analytically shown in Appendix A that indeed the star's surface must be entirely surrounded by corotating electrospheric plasma. These two charged regions are separated by large vacuum gaps. We experienced some difficulty in obtaining the exact shape of the border of domes near the null line because at this location $\rho_{\mathrm{GJ}}$ almost vanishes. As a result, any little numerical roundoff error in the charge contained in this flux tube, $q_{\mathrm{P}}$, significantly affects the estimation of $R_{\gamma}$. Some inner part of the equatorial belt is directly connected along field lines to the star without any gap, and is thus corotating with it, while the outer part, which is not electrically connected by field lines to the star is super-rotating everywhere. These results are in agreement with those of Rylov (1985). The differential Goldreich-Julian density is sharply peaked at the innermost part of this latter region but never exceeds three or four times the corotation value. We have also plotted the residual stellar surface charge in comparison to the initial one in order to illustrate the quality of our numerical results: at the end of the iteration process no more than $1 \%$ of the initial surface charge is left on the star's surface.

A second example, with $Q_{\text {tot }}=3 Q_{\mathrm{c}}$, is represented in Fig. 3. This value of the total charge corresponds to the limit case separating electrospheres of a finite extent $\left(Q_{\mathrm{tot}} \leq 3 Q_{\mathrm{c}}\right)$ from those which extend beyond the light cylinder $\left(Q_{\mathrm{tot}} \geq 3 Q_{\mathrm{c}}\right)$, and therefore escape our present non-relativistic description. As compared to the previous case, it can be seen that the domes have substantially shrunk and the disc has expanded because initially there were less electrons but more ions available on the stellar surface. Here again we reach the same conclusion as in the former case, namely that the star is entirely surrounded by its electrosphere. By contrast to the preceding model we encountered no difficulty in calculating its structure near the null line. The inner part of the equatorial belt is corotating with the star while the outer part is superrotating. The rotation rate reaches $2 \Omega_{*}$ at infinity.

The differences just noted illustrate a general trend. Indeed, beginning with a model with a low total charge, an increase in the value of $Q_{\text {tot }}$ shrinks the polar regions while it expands the equatorial disk, also causing an increase of the differential rotation rate. This is explained by the fact that filling up the belt increases the radially outward component of the electric field and causes a faster particle drift motion. As a consequence the rotation rate increases.

Another set of models has been computed, removing the dome. This means that negative surface charge has not been transfered from the star to the electrosphere, and therefore such models retain some surface charge at some places on the star's surface. We found that the structure of the equatorial region had not been drastically perturbed, though. We conclude that the domes have only a minor 

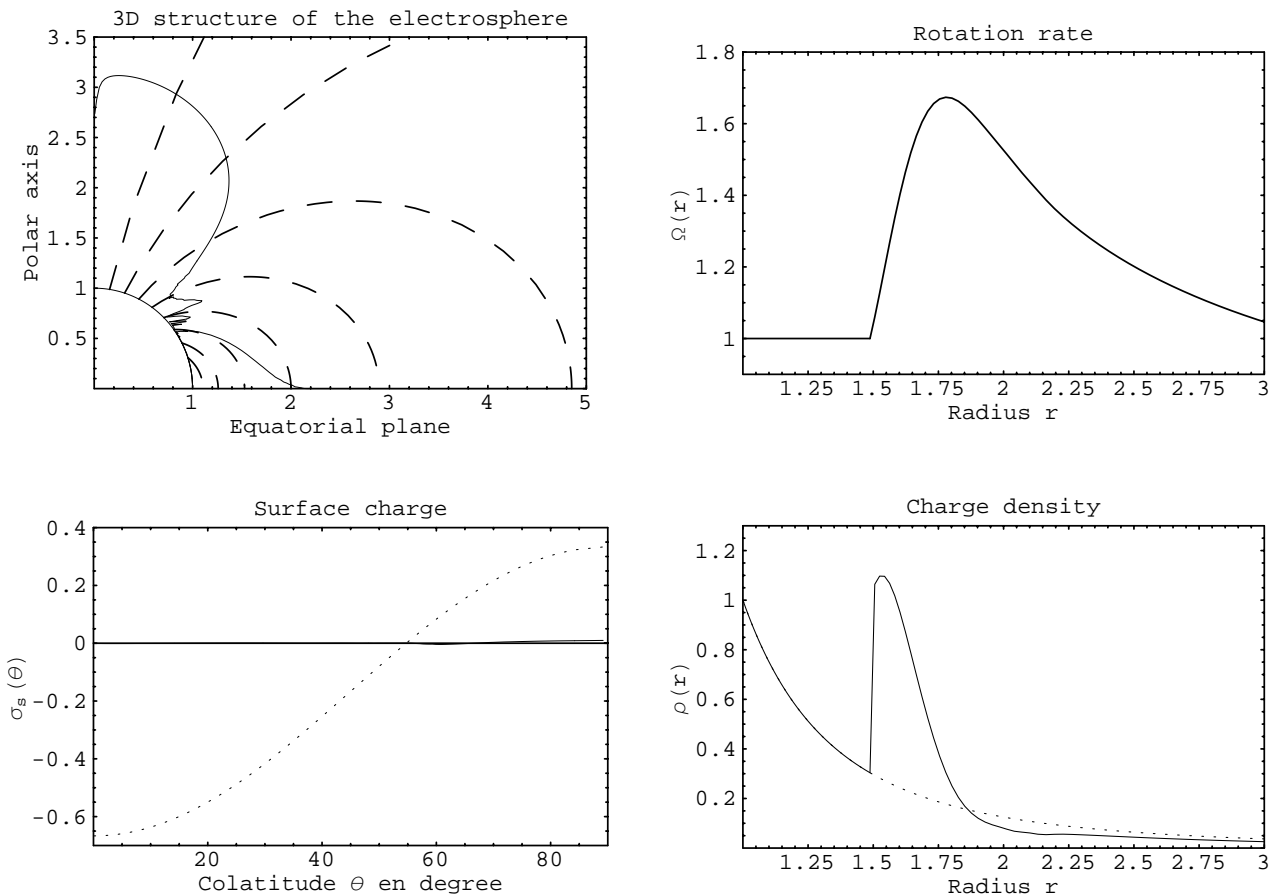

Fig. 2. Main characteristics of the electrosphere for the model with a total charge $Q_{\text {tot }}=Q_{\mathrm{c}}$. The figure on the top left shows its geometry, with the neutron star's surface depicted by the quarter-circle and the calculated boundaries of the positively charged equatorial belt and the negatively charged northern dome represented by full lines. The magnetic field lines are drawn in dashed. On the top right the rotation rate of the disk is plotted versus distance from the pulsar center. The corotation and differentially rotating regions appear clearly. On the bottom left, the residual stellar surface charge (solid line) is compared with the initial one (dotted line). It can be seen that at convergence negligible surface charge remains. On the bottom right the differential Goldreich-Julian density (solid line) is plotted as a function of distance from the pulsar center and compared to the corotating Goldreich-Julian density (dashed line).
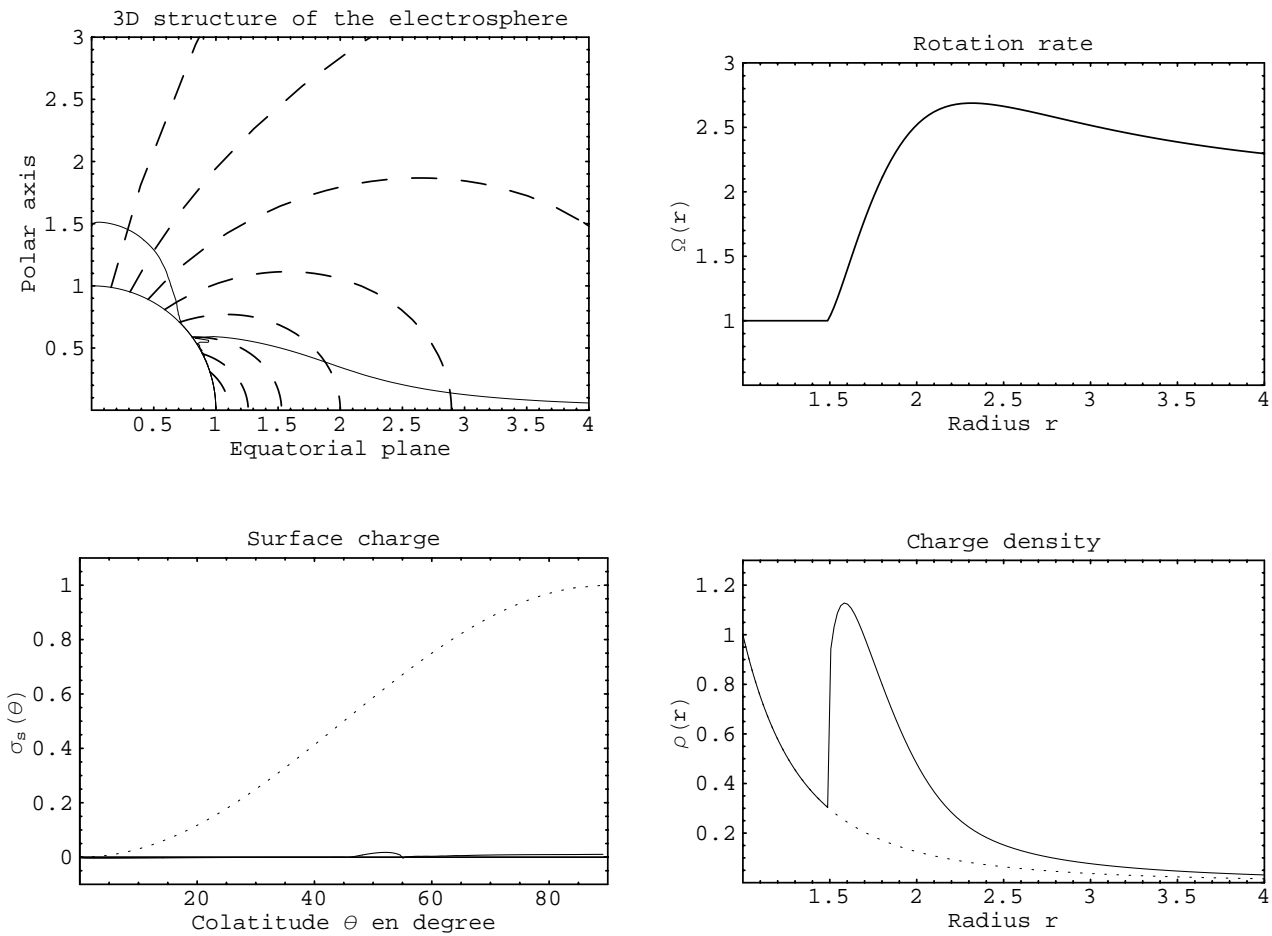

Fig. 3. Main characteristics of the electrosphere for the model with total charge $Q_{\mathrm{tot}}=3 Q_{\mathrm{c}}$. The quantities represented are the same as in Fig. 2. 
effect on the structure of the differentially rotating disk (domes only make it possible to bring more ions in the belt).

Replacing the dipolar magnetization by an uniform one in the pulsar makes it impossible to achieve convergence to any equilibrium state. A large amount of positive charge remains present on the surface due to a sharp discontinuity in the electric field component normal to the interface star-electrosphere, the complete disappearance of which appears to be impossible. This is due to the fact that the electric currents which generate the magnetic field are in this case flowing on the surface of the neutron star and not at a central singularity as in the case of a dipolar field. The electric charge density associated with these currents (see Eq. (23)) consequently has in this case a surfacic type of singularity on the star's surface which is inherent to this particular magnetic structure and cannot be removed.

It is important to stress that our solutions are independent of how charges are transfered from the star to the electrosphere. Indeed, different choices of transfer function, taking for example $p(\theta, \lambda)$ in Eq. (28) to depend linearly or quadratically on the surface charge density with different multiplicative factors have been tested to cause no change in the results shown above. So the solution to which our algorithm converges is unique. Indeed, as shown in Appendix B, the solution to the electrosphere structure is unique for a given value of $Q_{\text {tot }}$.

We emphasize the fact that in all these models the gaps are stable in the sense that if somehow a particle were to be found or created in the gap it would be attracted to the region of the electrosphere with the same charge sign as its own and repelled from the region of opposite sign. This is why gaps actually exist (Holloway 1973). So no electric current is to flow from one to the other charge-separated region, unless the system were to suffer large amplitude perturbations.

We summarize below the essential features of the results thus far obtained:

- large gaps separate the equatorial from the polar charge-separated plasma regions. The configuration of the equipotentials leaves no possibility for electric current to flow from domes to disc or conversely;

- the electrosphere is finite in extent as long as the total charge does not exceed three times the central point charge: $Q_{\text {tot }} \leq 3 Q_{\mathrm{c}}$. If moreover $Q_{\text {tot }} \ll 3 Q_{\mathrm{c}}$ the plasma is confined well within the light cylinder;

- the part of the equatorial belt which is electrically connected to the stellar surface (extending in the equatorial plane from $r=R_{*}$ to about $\left.r=1.5 R_{*}\right)$ as well as the domes are in corotation. For $r \geq 1.5 R_{*}$ the belt is super-rotating everywhere, reaching a maximum of several stellar rotation velocity in the vicinity of $r=2 R_{*}$ and then decreasing slowly;

- the maximum of the Goldreich-Julian density is in all cases the same. It never exceeds a few times the corotation Goldreich-Julian density. However its location is placed increasingly outwards with increasing total charge.

We show additionally in Fig. 4 the structure of the electrosphere with $Q=0$ which is very similar to those discussed in Fig. 2. The qualitative conclusions made above remain true. The disk has shrunk to a very tiny region in the vicinity of the equatorial plane. Although the dome is poorly delimited near the null-line because of numerical round-off error, it is clear that the dome doesn't extend to infinity, it reaches only $4.5 R_{*}$. This remark applies whatever the total charge $Q$ might be. Indeed, an infinite dome contains an infinite charge but in order to conserve the total charge of the system, the disk must then carry an oppositely infinite charge compensating the former so that the belt extends to infinity too, a situation which is not allowed due to the presence of the light surface.

\subsection{General relativistic effects}

The neutron star gravitational radius, $R_{\mathrm{g}}=G M_{*} / c^{2}$, is, for typical neutron star parameters, of order $R_{\mathrm{g}} \approx 0.15 R_{*}$ which is not much less than the star's radius. The spacetime is thus strongly curved in the neighborhood of the neutron star surface. This will alter the structure of the electromagnetic field as compared to our classical calculation. It has been shown that for a slowly rotating black hole metric (described by the Kerr metric expanded to first order in the stellar angular momentum $J_{*}$, which is a good approximation if $J_{*} / M_{*}$ is small) the correction in the magnetic field as compared to the Minkowski flat space-time case is small. The still dipolar field is increased in magnitude by roughly $10 \%$. To first order in $J_{*}$, only the electric field is enhanced by the inertial frame dragging effect (Rezzolla et al. 2001).

Adapting our algorithm to curved space-time would imply replacing the dipolar magnetic field by its general relativistic expression. The situation for an aligned rotator would remain axisymmetric. The electric field is to be found from Poisson's equation in curved space, which implies the determination of the corresponding Green's function. The Goldreich-Julian density is replaced by its general-relativistic analog, given by Muslimov \& Tsygan (1992) (see also Muslimov \& Harding 1997)

$$
\begin{aligned}
\rho_{\mathrm{GJ}}^{\mathrm{GR}} & =-\varepsilon_{0} \operatorname{div}[f(R)(\Omega \times R) \times B] \\
& =\left[f(R)+\frac{R \sin \theta}{2} \frac{\mathrm{d} f}{\mathrm{~d} R}\right] \rho_{\mathrm{GJ}} .
\end{aligned}
$$

The corrective factor $f(R)=\frac{1}{\sqrt{1-R_{\mathrm{g}} / R}}\left(1-\frac{R_{\mathrm{g}} I_{*}}{M_{*} R^{3}}\right) . I_{*}$ is the stellar moment of inertia. The charge density $\rho_{\mathrm{GJ}}$ is the classical non-general-relativistic Goldreich-Julian charge density calculated for the magnetic field structure associated to curved space. It differs from the classical one by a few percent, except very near the star.

For a uniform star mass density, the moment of inertia $I_{*}=\frac{2}{5} M_{*} R_{*}^{2}$. Then, for $R \geq R_{*}$ the factor $f$ is very 

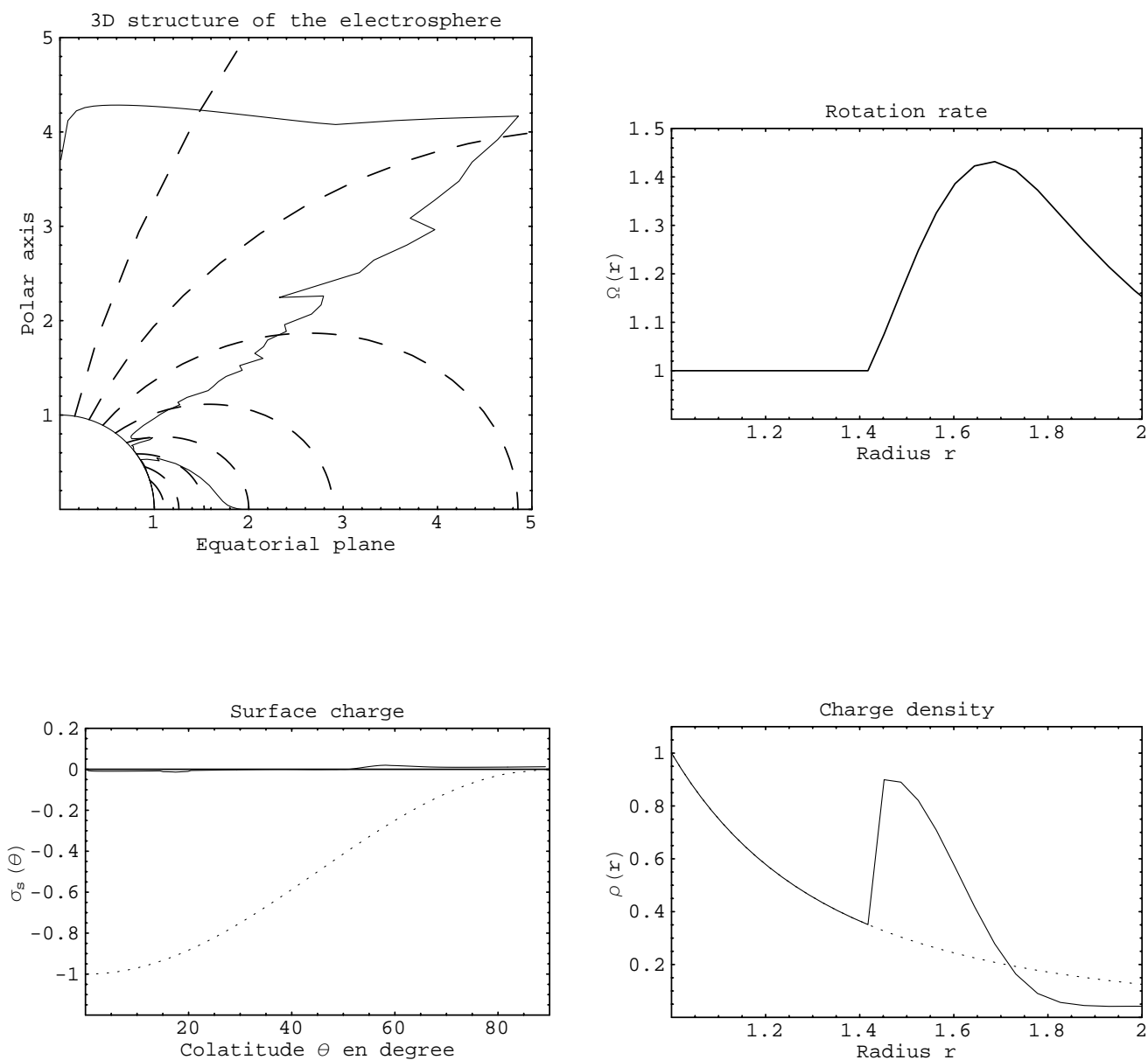

Fig. 4. Main characteristics of the electrosphere for the model with null total charge $Q_{\text {tot }}=0$. The quantities represented are the same as in the previous figures. Note that the dome doesn't extend to infinity.

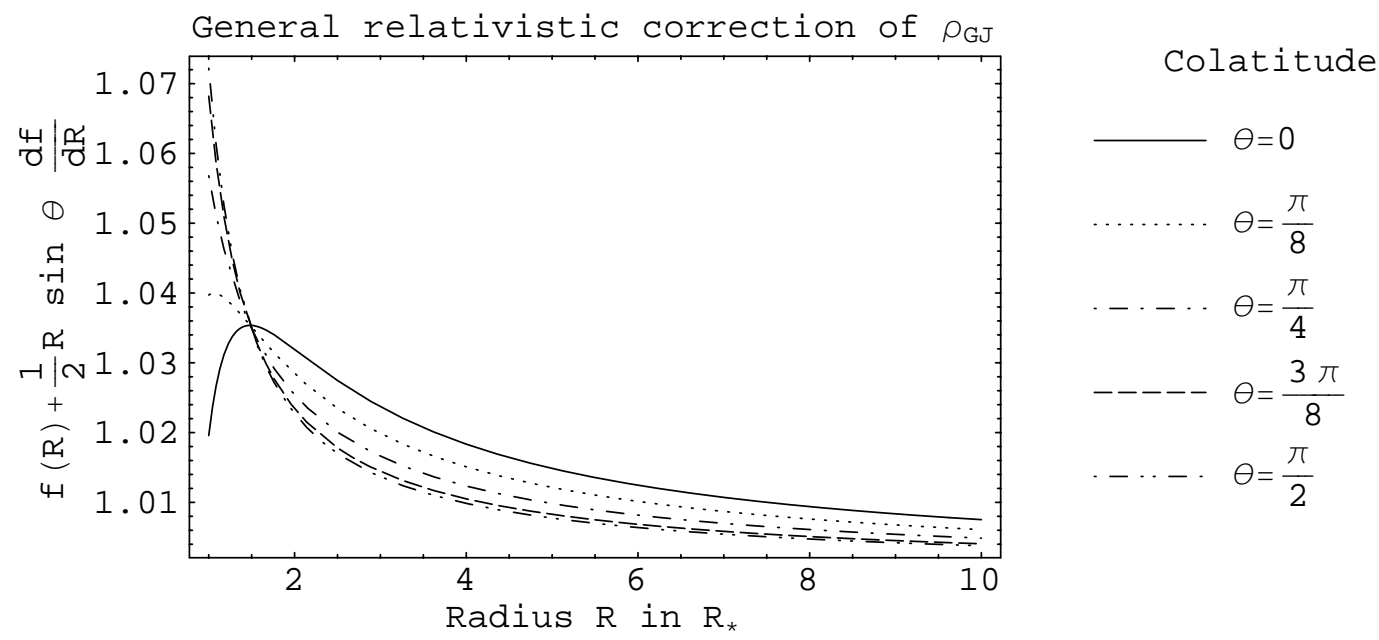

Fig. 5. Correction coefficient $f(R)+\frac{R \sin \theta}{2} \frac{\mathrm{d} f}{\mathrm{~d} R}$ for the Goldreich-Julian charge density.

close to unity and the first derivative $R \mathrm{~d} f / \mathrm{d} R$ never exceeds 0.1 . The function $f(R)+\frac{R \sin \theta}{2} \frac{\mathrm{d} f}{\mathrm{~d} R}$ is plotted in Fig. 5 for different values of the colatitude $\theta$. The charge density is in fact not significantly affected by curved space-time, except very near to the neutron star surface in the equatorial plane. In an equilibrium state, this charge density still screens the component of the electric field parallel to the magnetic field line and no particle acceleration occurs.

Therefore the qualitative kinematic and geometric characteristics of the electrosphere is not altered by the general relativistic considerations of an electromagnetic field in curved space-time. The results obtained above are 

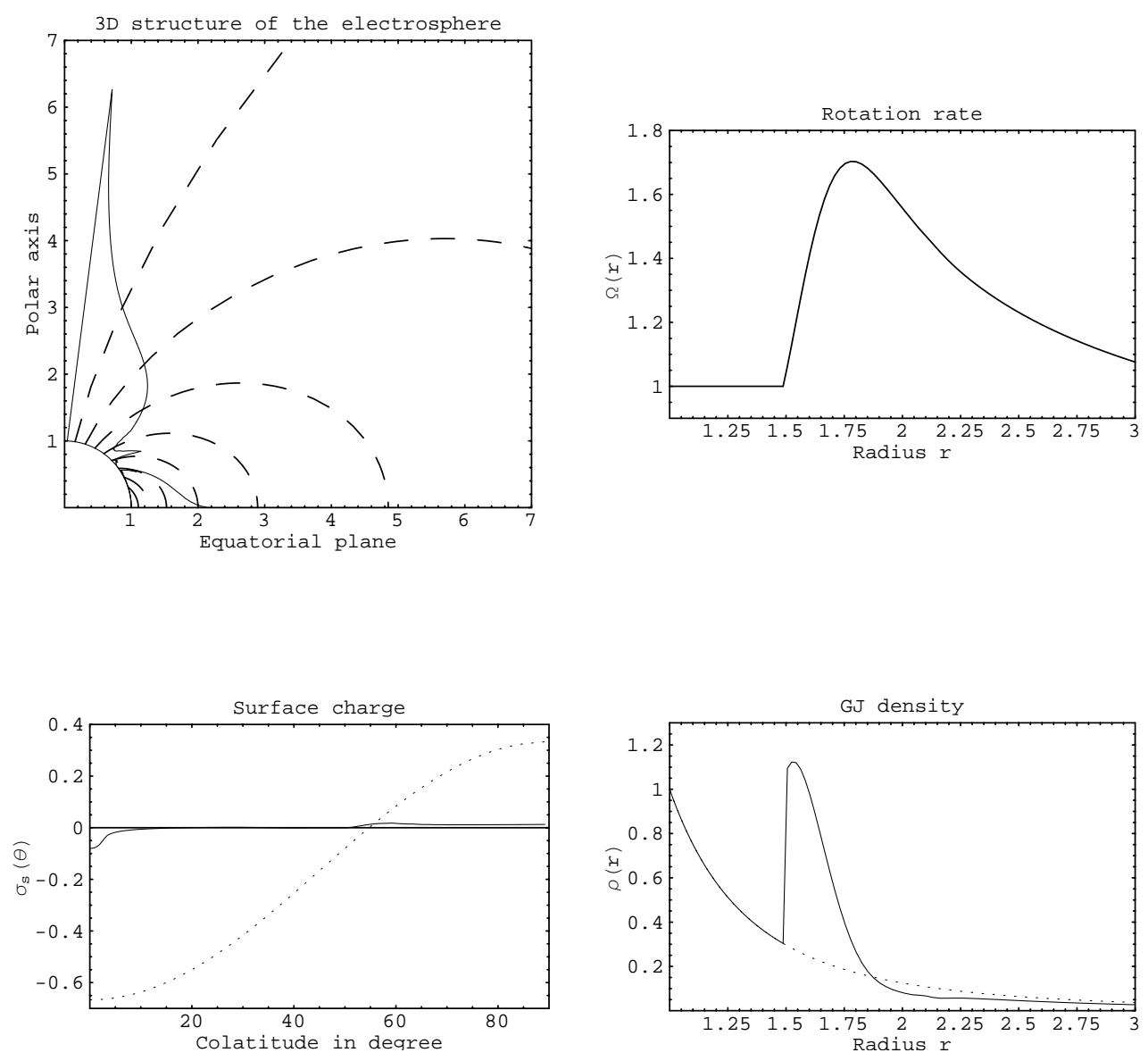

Fig. 6. Main characteristics of the electrosphere for a model with net charge loss from the polar caps when the total charge has reached the value $Q_{\text {tot }}=Q_{\mathrm{c}}$. The size of the polar cap corresponds to the region of open magnetic field lines in the case of a second pulsar. The particles extracted from the small circular polar caps supposedly escaped to infinity. Negative surface charge always persists at the polar cap. The quantities represented are the same as in Fig. 2, to which these results may be compared.

quantitatively accurate to a few percent as can be judged from Fig. 5 .

\subsection{Charge loss from the polar caps}

The main consequence of the existence of light cylinder effects would be to induce a net loss of charge from the polar caps by a charged wind. This is possible in a stationary state only if return currents can flow in the pulsar's environment. If such currents can not develop, either temporarily or on the long term, charge loss will cause an increase of the total charge. In order to simulate in a simple way such an effect we have altered our algorithm as follows. Instead of transferring any surface charge to the electrosphere, we did so only for those regions on the star which are magnetically linked to regions interior to the light cylinder. Surface charge extracted from the polar caps is rejected to infinity instead of being stored in the electrosphere, at least as long as this remains consistent with the sign of the potential difference between the star and infinity. This should simulate accurately enough the effect on the electrosphere of charge loss. Indeed we have seen above that the structure of the differentially rotating electrospheric disc is hardly sensitive to the structure of the domes. Figures 6 and 7 show the structure of the plasma region with such a polar charge loss. These results are to be compared with those corresponding to models without charge escape presented in Figs. 2 and 3.

From this, three remarks are to be made. First, as expected, the global shape and characteristics of the closed electrosphere are not much altered by including such a charge loss process. The disk differential rotation rate, for example, remains essentially unaltered. Secondly, only negative surface charge appears at the polar caps during the transfer process, so that the wind remains exclusively composed of particles of a common and same (negative) sign. From this it results that the net total charge $Q_{\text {tot }}$ of the star and electrosphere system steadily increases with the number of iteration steps. Indeed, from Fig. 8, it clearly appears that $Q_{\text {tot }}$ increases roughly linearly with the iteration number, the net loss per step becoming constant, although very small and significantly lower than the total charge (only $\approx 10^{-6}$ of $Q_{\text {tot }}$ ). Third, we note that this increasing total charge will eventually prevent any subsequent particle escaping for some value of $Q_{\text {tot }}$ of order of $Q_{\mathrm{c}}$.

In order to give a more accurate estimation of this value we have to distinguish two inherently different 

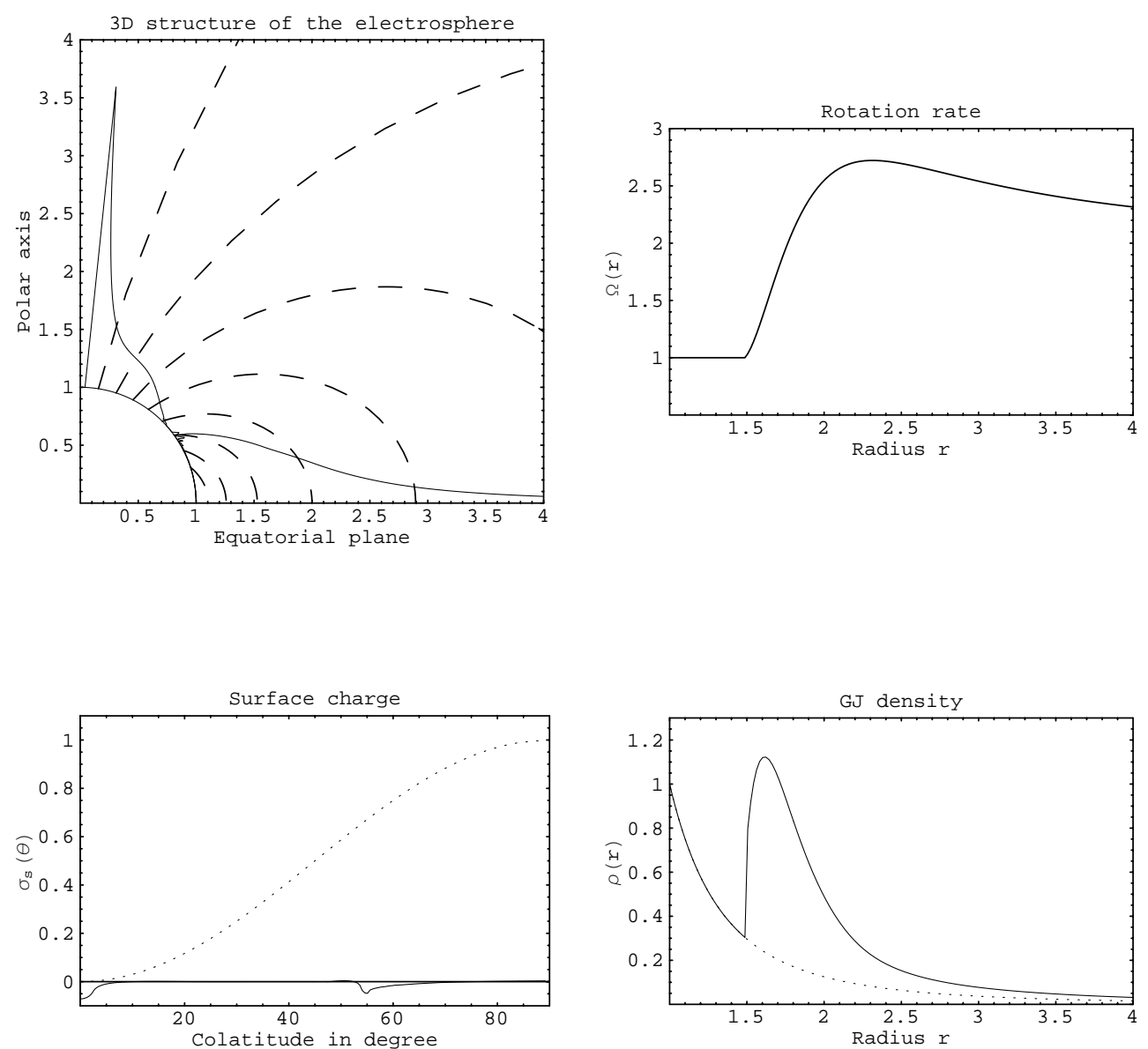

Fig. 7. Main characteristics of the electrosphere for a model with net charge loss from the polar caps when the total charge has reached the value $Q_{\mathrm{tot}}=3 Q_{\mathrm{c}}$. The particles extracted from the small circular polar caps supposedly escaped to infinity. The quantities represented in the figures are the same as in Fig. 3 to which these results may be compared.
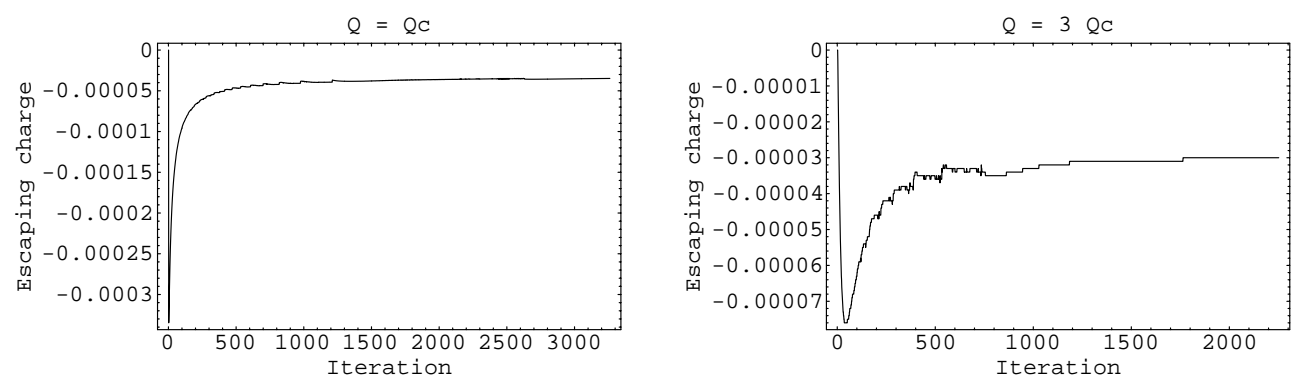

Fig. 8. Charge escaping from the polar caps for the model $Q=Q_{\mathrm{c}}$ (on the left) and $Q=3 Q_{\mathrm{c}}$ on the right. The charge lost per iteration becomes very small and eventually remains almost constant for the 2 cases. This indicates that the total net charge of the system increases as iteration goes on.

scenarios which differ by the initial net charge. Let's imagine that we begin by taking this charge to be null (the same consequences would hold for negative net charge) which implies that only negative particles appear on the stellar surface. These ones will induce a wind emanating from the two polar caps with a negative net outgoing flux as mentioned above. As time goes, $Q_{\text {tot }}$ increases until the potential drop between star and infinity becomes unfavorable for charge escape (i.e. vanishes). The simulations have shown that this occurs for $Q_{\mathrm{tot}}=Q_{\mathrm{opt}}^{<} \approx Q_{\mathrm{c}}$. From then on, it becomes possible to fill the hollow cones with trapped negative particles and to reconstruct an entire dome reaching the polar line. Due to the circulation of charge via the neutron star surface, the domes will shrink and transfer some charge into the empty polar region. As a result we turn back to the static electrosphere described in the preceding section but now with a total charge of the system imposed by the electrosphere itself.

If now we take an initial total charge $Q_{\text {tot }} \geq 3 Q_{\mathrm{c}}$, the situation is reversed, positive particles present at the polar 
caps are effectively extracted from the crust and reach infinity because of the favorable potential drop. This positive net outgoing charge flux decreases $Q_{\text {tot }}$ until it reaches a value $Q_{\mathrm{opt}}^{>} \approx 3 Q_{\mathrm{c}}$. At this stage, negative charge appears gradually at the pole, extending from the polar line to the null line (where $\rho_{\mathrm{GJ}}=0$ ), their extraction remains possible but the potential drop prevents the formation of any wind consisting of those particles. Here again static domes can develop and the electrospheric structure eventually looks like those shown in Fig. 3.

This leads to the conclusion that no stationary regime of particle escape can be met in which no net positive or negative charge loss occurs and that the final charge of the system stabilizes at $Q_{\text {opt }}^{</>}$depending on the system's initial charge. The exact value of $Q_{\text {opt }}$ is determined by the precise electrospheric configuration around the pulsar. This can easily be seen by expressing the condition for wind disappearance when equaling the polar line potential at the crust given by (8) for $a=0$ with its vanishing value at infinity. Doing this we get the optimal charge as

$Q_{\mathrm{opt}}=Q_{\mathrm{c}}+Q_{\mathrm{el}}+Q_{\text {imel }}$

Jackson (1979) showed that this total net charge minimizes the configurational potential energy of the electrosphere.

\section{Stability aspects}

Our description is only valid if the gaps present in our solutions are stable to charge transfer from disk to domes and to vacuum breakdown by pair avalanches. We stressed already their stability with respect to charge transfer. We now examine in some more detail the mechanism of $\mathrm{e}^{ \pm}$ pair creation in these outer gaps and show that the development of leptonic cascades in second pulsars with period of the order of $1 \mathrm{~s}$ or so is rather unlikely and certainly unable to cause the filling up of the magnetosphere with electron-positron plasma up to the light cylinder. We recall that the probability $p(l)$ of conversion into a lepton pair of a photon of energy $\hbar \omega$ along a path of length $l$ is given according to Erber (1966) by

$p(l)=1-\mathrm{e}^{-\int_{0}^{l} \alpha(\chi) \mathrm{d} s}$

where

$\alpha(\chi)=\frac{\alpha_{\mathrm{sf}}}{2 \lambda_{\mathrm{c}}} \frac{B_{\perp}}{B_{\mathrm{Q}}} T(\chi)$

is the attenuation coefficient, $\alpha_{\text {sf }}=\mathrm{e}^{2} / 4 \pi \varepsilon_{0} \hbar c$ the fine structure constant, $\lambda_{\mathrm{c}}=\hbar / m_{\mathrm{e}} c$ the Compton length of the electron, $B_{\perp}$ the magnetic field perpendicular to the photon path, $B_{\mathrm{Q}}=m_{\mathrm{e}}^{2} c^{2} / e \hbar$ the quantization magnetic field, $\chi$ the quantity

$\chi=\frac{\hbar \omega}{2 m_{\mathrm{e}} c^{2}} \frac{B_{\perp}}{B_{\mathrm{Q}}}$

and the function $T(\chi)$ is:

$T(\chi)=\frac{0.16}{\chi} K_{1 / 3}^{2}\left(\frac{2}{3 \chi}\right)$ where $K_{1 / 3}$ is the modified Bessel function of the second kind of order 1/3 (Abramowitz \& Stegun 1965). For a given photon energy, the attenuation coefficient is an increasing function of the perpendicular magnetic field component. So the main contribution to the disintegration probability comes from the regions of most intense magnetic field strength.

\subsection{Avalanches from curvature radiation photons}

Suppose that an electron (may be a positron) emits by curvature radiation a photon of energy $E_{\gamma}$ parallel to the local magnetic field at a some place on the null surface (where magnetic field and rotation speed are orthogonal), i.e at the position $\left(R_{0}, \theta_{0}=\arccos \frac{1}{\sqrt{3}}\right)$. When reaching a central distance $R$ during its trip, started at the emission point $R_{0}$, the photon sees a magnetic field $B_{\perp}$ perpendicular to its path given by $R^{3} B_{\perp}=B_{*} R_{*}^{3} P_{2}\left(\frac{R_{0}}{R} \cos \theta_{0}\right)$. For a dipolar magnetic field, the curvilinear abscissa along the photon path is related to the spherical radius by $R^{2}=R_{0}^{2} \cos ^{2} \theta_{0}+\left(s+R_{0} \sin \theta_{0}\right)^{2}$. So we get

$\mathrm{d} s=\frac{\mathrm{d} R}{\sqrt{1-\frac{R_{0}^{2}}{3 R^{2}}}}$.

Then the probability (31) depends on the photon energy $E_{\gamma}$, on the location of its emission $R_{0}$ and on the distance $l$ as:

$p\left(E_{\gamma}, R_{0}, l\right)=1-\mathrm{e}^{-\int_{0}^{l} \alpha\left(\chi\left(E_{\gamma}, R_{0}, R\right)\right) \mathrm{d} s(R)}$.

A good estimate of the free path length of this photon to pair creation is the length $l$ for which the conversion rate becomes close to unity. In other words, $l$ is the abscissa $s$ for which the probability of disintegration equals 0.999 for example, that is

$p\left(E_{\gamma}, R_{0}, l\right)=0.999$

Figure 10 shows the behavior of $l$ as a function of $E_{\gamma}$ and $R_{0}$ in the case of second and millisecond pulsars with typically $B_{*}=10^{8} \mathrm{~T}$ for the former and $B_{*}=10^{5} \mathrm{~T}$ for the latter. We also compute the corresponding optical depth useful for future comparison with the $\gamma-\gamma$ pair creation process (see 5.3). The optical depth to pair creation by photon interaction with the magnetic field is:

$\tau\left(E_{\gamma}, R_{0}\right)=\int_{0}^{\infty} \alpha\left(\chi\left(E_{\gamma}, R_{0}, R\right)\right) \mathrm{d} s(R)$

It is shown in Fig. 11 for various photon energies and emission positions.

Up to now the photon energy has been left unprecised. However we know that the synchro-curvature radiation spectrum peaks near the frequency $\omega=\frac{3}{2} \frac{\mathrm{c}}{R_{\mathrm{c}}} \gamma^{3}, R_{\mathrm{c}}$ being the curvature radius of the lepton's trajectory. The Lorentz factor $\gamma$ of the emitting electron cannot exceed the electric potential drop available in the gap on the magnetic surface on which it moves. Then we find as an upper bound $\gamma \leq e \delta \phi / m_{\mathrm{e}} c^{2}, \delta \phi(a)$ being the potential 

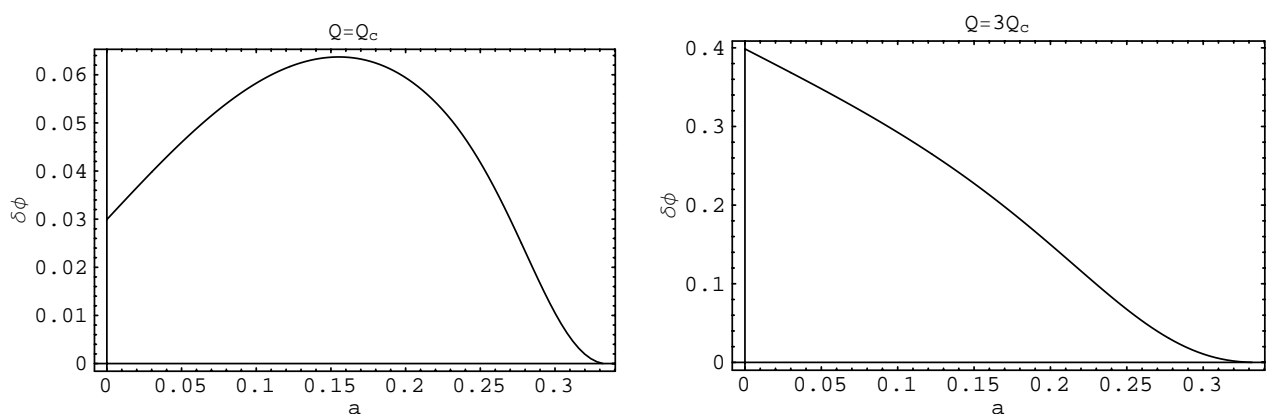

Fig. 9. The variation with magnetic line of the potential drop $\delta \phi$ established along it between a polar dome and the equatorial belt is represented for the model with initial total charge $Q_{\mathrm{tot}}=Q_{\mathrm{c}}$ (on the left) and $Q_{\mathrm{tot}}=3 Q_{\mathrm{c}}$ (on the right). The variable representing magnetic lines is their flux variable, a normalized to its equatorial value. The maximal potential drop is close to 0.06 in units of $\Omega_{*} B_{*} R_{*}^{2}$ for the former and 0.4 for the latter.
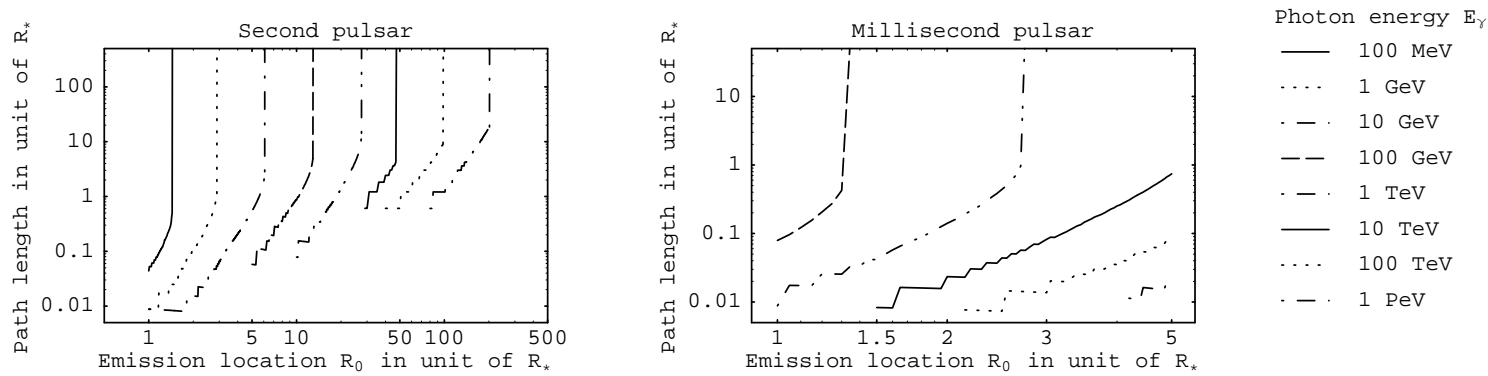

Fig. 10. Maximum path length before disintegration (defined by Eq. (37)) of a photon of a given energy $E_{\gamma}$ (indicated by the graphical code on the r.h.s, and ranging between $100 \mathrm{MeV}$ and $1 \mathrm{PeV}$ ) into an $\mathrm{e}^{ \pm}$pair in the dipolar magnetic field of a pulsar against the position of photon emission $R_{0}$ : on the left for a second pulsar $\left(B_{*}=10^{8} \mathrm{~T}\right)$ and on the right for a millisecond pulsar $\left(B_{*}=10^{5} \mathrm{~T}\right)$. Each curve represents the path length for a given photon energy. For periods of the order of a second no photon disintegration takes place for $1 \mathrm{MeV}$ and $10 \mathrm{MeV}$. Their corresponding curves are not represented. For millisecond pulsars the same apply for energies ranging from $1 \mathrm{MeV}$ to $10 \mathrm{GeV}$, which are not represented too.

drop between one dome and the equatorial belt along the magnetic line $a$, that is:

$\delta \phi(a)=\int_{a}^{\frac{1}{3} B_{*} R_{*}^{2}}\left(\Omega\left(a^{\prime}\right)-\Omega_{*}\right) \mathrm{d} a^{\prime}$.

Figure 9 shows how this potential drop varies with the flux variable $a$. It increases sharply in the vicinity of the star and reaches an almost constant value of about one tenth of the characteristic potential. Thus, except very near the star's surface, the gap supports a potential drop of or$\operatorname{der} \Omega_{*} B_{*} R_{*}^{2} / 10$. Consider a typical pulsar with a period in the range of a second (a second-pulsar). Let us adopt as representative values $B_{*}=10^{8} \mathrm{~T}, \Omega_{*}=2 \pi \mathrm{rad} / \mathrm{s}$ and $R_{*}=10 \mathrm{~km}$. Parameters appropriate for a millisecond pulsar are $B_{*}=10^{5} \mathrm{~T}, \Omega_{*}=2 \pi 10^{3} \mathrm{rad} / \mathrm{s}$ and $R_{*}=10 \mathrm{~km}$. In both cases, the potential drop would produce accelerated particles with a Lorentz factor of order $\gamma \approx 10^{11}$. This value is however a gross overestimate of the lepton's Lorentz factor $\gamma$, because the radiation reaction imposes more rigorous constraints. Equating the power lost by curvature radiation $P=\frac{e^{2}}{6 \pi \varepsilon_{0} c^{3}} \gamma^{4} \Gamma^{2}$ (where $\Gamma=c^{2} / R_{\mathrm{c}}$ is the particle acceleration) with that provided by the electric field, $e \boldsymbol{E} \cdot \boldsymbol{v}$, we obtain an estimate of the energy reached by the particles with consideration of radiation reaction effects,

$\gamma_{\max }=\left(\frac{6 \pi \varepsilon_{0}}{e} R_{\mathrm{c}}^{2} E_{\max }\right)^{\frac{1}{4}}$

where $E_{\max }$ is the maximum value of the electric field. For both second or millisecond pulsars, taking $R_{\mathrm{c}}=10 R_{*}$ and $E_{\max }=\Omega_{*} B_{*} R_{*}$, we find $\gamma_{\max } \approx 10^{8}$.

The energy of curvature photons never exceeds that of their emitter, which appears to be limited to approximately $10^{8} m_{\mathrm{e}} c^{2}$. In fact most curvature photons are much less energetic than the lepton which emits them so that $E_{\gamma} \ll 10^{8} m_{\mathrm{e}} c^{2}$. The characteristic curvature photon energy is given by $E_{\gamma}^{\mathrm{c}}=\frac{3}{2} \hbar \frac{c}{R_{\mathrm{c}}} \gamma_{\max }^{3}$. With $R_{\mathrm{c}}=10 R_{*}$ we find $E_{\gamma}^{\mathrm{c}} \approx 3 \mathrm{TeV}$.

Looking at Fig. 10, we see that for pulsars with a magnetic field strength of $B_{*}=10^{8} \mathrm{~T}$ the breakdown region is limited to about fifty stellar radii (independently of his rotation period). This is negligible compared to the light cylinder radius for both $P=1 \mathrm{~s}\left(R_{\mathrm{L}} \approx 4800 R_{*}\right)$ and $P=0.1 \mathrm{~s}\left(R_{\mathrm{L}} \approx 480 R_{*}\right)$. This only goes to show that curvature photon conversion into a pair on the magnetic field is not an efficient enough mechanism to break the vacuum gaps which appear in our solutions. Unlike secondpulsars, millisecond-pulsars could fill their vacuum gaps with pair plasma up to the light surface although their 

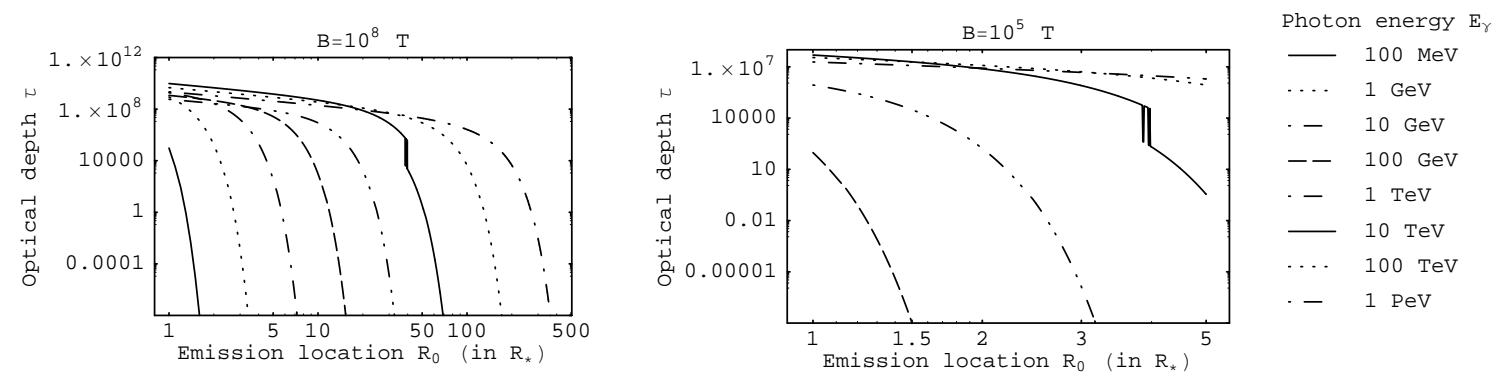

Fig. 11. Optical depth $\tau$ as obtained by Eq. (38). Exactly the same remarks are to be made as in the legend of Fig. 10.

magnetic field is only $B_{*}=10^{5} \mathrm{~T}$. Indeed, looking at the panel on the right of Fig. 10, it is seen that the extension of the vacuum breakdown zone for very energetic photons of about 0.1 to $1 \mathrm{TeV}$ and more, is of the same order of magnitude magnitude as the light cylinder radius $R_{\mathrm{L}}$, about $5 R_{*}$. We conclude that a magnetosphere entirely filled with leptonic plasma is quite possible in this case.

\subsection{Inverse Compton scattering}

This statement remains true if the hard photons were to originate from inverse Compton scattering of soft photons by ultra-relativistic electrons or positrons rather than from curvature radiation. The maximal energy reached by such photons is obviously less than the total kinetic energy of the energetic electron involved in the scattering process, the Lorentz factor of which is itself limited by curvature radiation reaction to a value of magnitude $10^{8}$. The scattered photon energy $E_{\gamma}$ never exceeds $100 \mathrm{TeV}$. Consequently, according to Fig. 10, the same conclusions as in the previous subsection hold.

\subsection{Pair production by $\gamma-\gamma$ interaction}

The two-photon pair production is generally considered in outer gap models as a trigger for $\mathrm{e}^{-} \mathrm{e}^{+}$cascade. Curvature gamma rays emitted by accelerated particles interact with the thermal X-ray photons from the polar caps. We show in this section that, for sufficiently low surface temperature, this process is negligible in the pulsar magnetosphere.

For convenience, in the following, the photon energy will be expressed in units of $m_{\mathrm{e}} c^{2}$. The threshold energy $\varepsilon_{\mathrm{s}}$ of the soft X-ray photon for pair creation by collision with a photon of energy $\varepsilon_{\gamma}$ at an angle $\zeta$ is then given by

$\varepsilon_{\mathrm{s}}=\frac{2}{1-\cos \zeta} \frac{1}{\varepsilon_{\gamma}}$.

For black body radiation from a small polar cap at a temperature $T$, the soft photon density per unit energy band at a distance $R$ is (Hirotani 2000)

$n_{\varepsilon_{x}}(T, R)=\frac{1}{4 \pi^{2} \lambda_{\mathrm{c}}^{3}} \frac{\varepsilon_{x}^{2}}{\exp \left(\varepsilon_{x} / \Delta\right)-1} \frac{A_{\mathrm{s}}}{4 \pi R^{2}}$

where $A_{\mathrm{S}}=\pi R_{*}^{3} / R_{\mathrm{L}}$ corresponds to one polar cap emission area, $\Delta=k_{\mathrm{B}} T / m_{\mathrm{e}} c^{2}$ and $\lambda_{\mathrm{c}}=\hbar / m_{\mathrm{e}} c$ is the electron

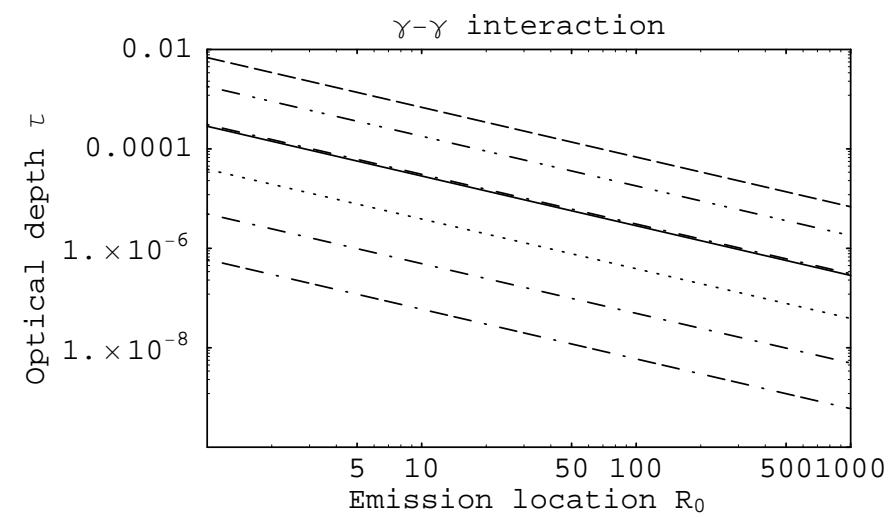

Fig. 12. Optical depth $\tau$ vs. emission location $R_{0}$ of the gamma-ray for $\varepsilon_{\gamma}=210^{j}$ with $j=3 \ldots 9$ and neutron star surface temperature $T=10^{6} \mathrm{~K}$. The slope of the curve is exactly $(-1)$ which means that $\tau R_{0}=$ cste.

Compton length. Inwardly propagating hard $\gamma$-photons are the most likely ones to interact with this background thermal emission. So we focus on them. Their mean free path $\lambda$ at the position $(R, \theta)$ is independent of $\theta$ and given by

$\frac{1}{\lambda}=\int_{\varepsilon_{\mathrm{s}}}^{\infty} n_{\varepsilon_{x}}(T, R)(1-\cos \zeta) \sigma_{\gamma \gamma}(\beta) \mathrm{d} \varepsilon_{x}$.

The pair production cross section in the zero-momentum frame, $\sigma_{\gamma \gamma}$, is given by

$$
\begin{aligned}
\sigma_{\gamma \gamma}(\beta)= & \frac{\pi}{2} r_{\mathrm{e}}^{2}\left(1-\beta^{2}\right)\left[\left(3-\beta^{4}\right) \ln \left(\frac{1+\beta}{1-\beta}\right)\right. \\
& \left.-2 \beta\left(2-\beta^{2}\right)\right]
\end{aligned}
$$

where $r_{\mathrm{e}}=\mathrm{e}^{2} / 4 \pi \varepsilon_{0} m_{\mathrm{e}} c^{2}$ is the electron classical radius and

$\beta=\sqrt{1-\frac{2}{1-\cos \zeta} \frac{1}{\varepsilon_{x} \varepsilon_{\gamma}}}$.

The optical depth for photon-photon collision is therefore

$$
\begin{aligned}
\tau & =\int_{R_{0}}^{R} \frac{\mathrm{d} s}{\lambda} \\
& =\int_{R_{0}}^{R} \int_{\varepsilon_{\mathrm{s}}}^{\infty} n_{\varepsilon_{x}}(T, R)(1-\cos \zeta) \sigma_{\gamma \gamma}(\beta) \mathrm{d} \varepsilon_{x} \mathrm{~d} s .
\end{aligned}
$$

The factor $(1-\cos \zeta)$ comes from the transformation from the zero-momentum frame to the laboratory frame. 


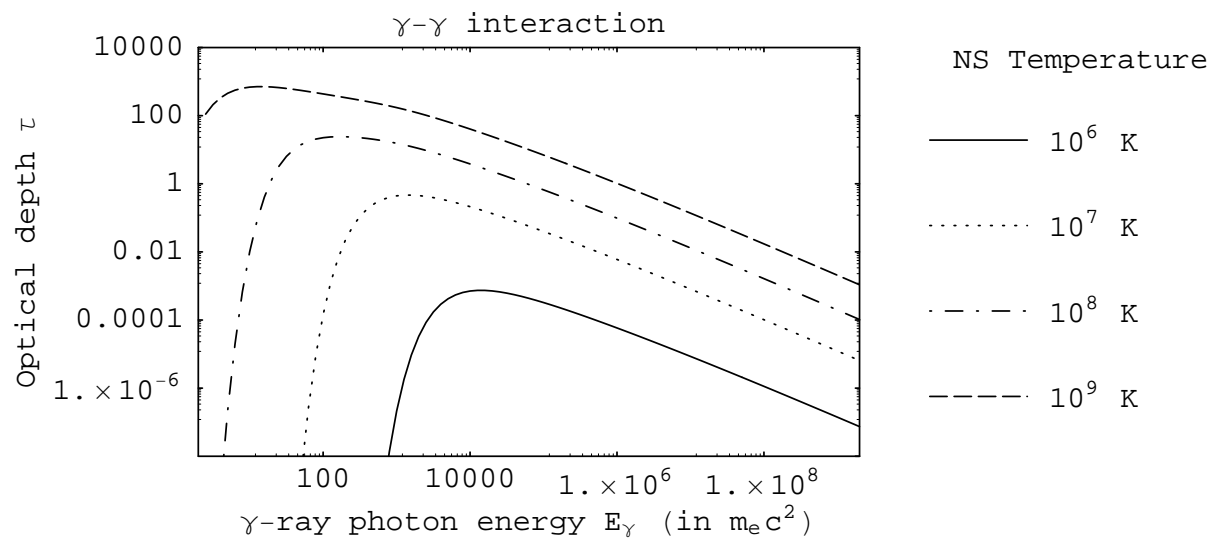

Fig. 13. Optical depth $\tau$ vs. gamma photon energy $\varepsilon_{\gamma}$ for different neutron star temperatures and for an emission location $R_{0}=$ $10 R_{*} . \tau$ increases with the neutron star temperature $T$. Realistic values of $T$ are $10^{6} \mathrm{~K}$ and $10^{7} \mathrm{~K}$. This optical depth is to compared with the one obtained by the photon disintegration in the magnetic field Fig. 11 . The polar cap area $A_{\mathrm{s}}$ is calculated with $R_{\mathrm{L}}=5000 R_{*}$ corresponding to a second pulsar. For a millisecond one, the optical depth is $10^{3}$ times greater.

For photons emitted at the null surface and propagating towards the axis, the relation between curvilinear abscissa and spherical distance is given by

$\mathrm{d} s=-\frac{\mathrm{d} R}{\sqrt{1-\frac{R_{0}^{2}}{3 R^{2}}}}$.

The minus sign reflects inward propagation of the photon. The collision angle $\zeta$ is given by

$\cos \zeta=-\sqrt{1-\frac{R_{0}^{2}}{3 R^{2}}}$

Numerical integration of the double integral in Eq. (46) has been made. The optical depth is easily seen to be inversely proportional to the distance to the star of the location of the gamma ray emission $\left(R_{0}\right)$. This scaling allows us to calculate $\tau$ for some reference $R_{0}$ only. Figure 13 represents the optical depth as a function of the gamma-ray energy $\varepsilon_{\gamma}$ and the neutron star temperature $T$. The emission of the curvature gamma photon has been assumed to take place at $R_{0}=10 R_{*}$. The upper limit of the radial integral is placed at the intersection of the photon path with the polar axis, at $R=R_{0} / \sqrt{3}$. Thus the integral is performed in the interval $\left[R_{0} / \sqrt{3}, R_{0}\right]$.

The optical depth sharply increases with energy up to a certain maximum and then decreases more slowly with increasing photon energy (see Fig. 13). For instance, for $T=10^{6} \mathrm{~K}$, the maximal optical depth, $\tau=6 \times 10^{-4}$, is reached for $\varepsilon_{\gamma}=10^{4} m_{\mathrm{e}} c^{2}$. Near the light cylinder of a pulsar-second, this depth is 100 times less, due to the $1 / R_{0}$ scaling. It is then clear that no two-photon interaction takes place for such a temperature, whatever the gamma-ray energy and its emission location. This remains true for $T=10^{7} \mathrm{~K}$, the optical depth reaching 0.4 for $\varepsilon_{\gamma}=1600 m_{\mathrm{e}} c^{2}$. For $T=10^{8} \mathrm{~K}$, however, the maximal optical depth reaches 24 for $\varepsilon_{\gamma}=160 m_{\mathrm{e}} c^{2}$ and this process becomes important.

Comparing the optical depths in Figs. 11 and 13, it is seen that photon-photon interaction is negligible compared to photon disintegration in the magnetic field.
Thus the $\gamma-\gamma$ interaction remains inefficient for sufficiently low neutron star temperatures of the order of $T=$ $10^{6} \mathrm{~K}$ or $T=10^{7} \mathrm{~K}$. It becomes significant for a hot stellar crust with $T \approx 10^{8} \mathrm{~K}$ and even more so for very hot ones. Such very high temperatures are however not likely to be met. For usual surface temperature (less than $10^{7} \mathrm{~K}$ say), the process of lepton pair creation by photon-photon collisions is dominated by the photon interaction with the magnetic field very near the neutron star.

\subsection{Consequence for the electrospheric equilibrium structure}

In the previous sections, we have shown that pair creation does not take place very far away from the star for second-pulsars. Indeed, given that the characteristic curvature photon energy is about a $\mathrm{TeV}$, the avalanche zone does not exceed a few tens of stellar radii (say $20 R_{*}$ with $B=10^{8} \mathrm{~T}$ ). How does this process influence the electrospheric structure? Qualitatively, we can answer this question as follows. As long as the gaps remain large enough to sustain a sufficient potential drop between disc and domes to create pairs, the equatorial belt will be filled with positrons (for aligned rotators) and the domes with electrons. The size of the gap will gradually diminish until the potential drop falls below the breakdown potential. Its dimension will become negligible as compared to the stellar radius. Thus in those regions where gaps become small, we may simply assume that the domes and the disc are in contact at the null surface and the corresponding magnetic surfaces are in corotation with the star. These surfaces, where the pair-cascade occurred, are almost entirely filled with charge-separated plasma at the Goldreich-Julian density. The gaps become eventually inactive and the new electrospheric structure will the be similar to the structure calculated in the absence of pair creation, simply changing the stellar radius $R_{*}$ to a new "effective" one, almost equal to the extension $R_{\mathrm{p}}$ of the avalanche zone. From an electrical point of view, there is 

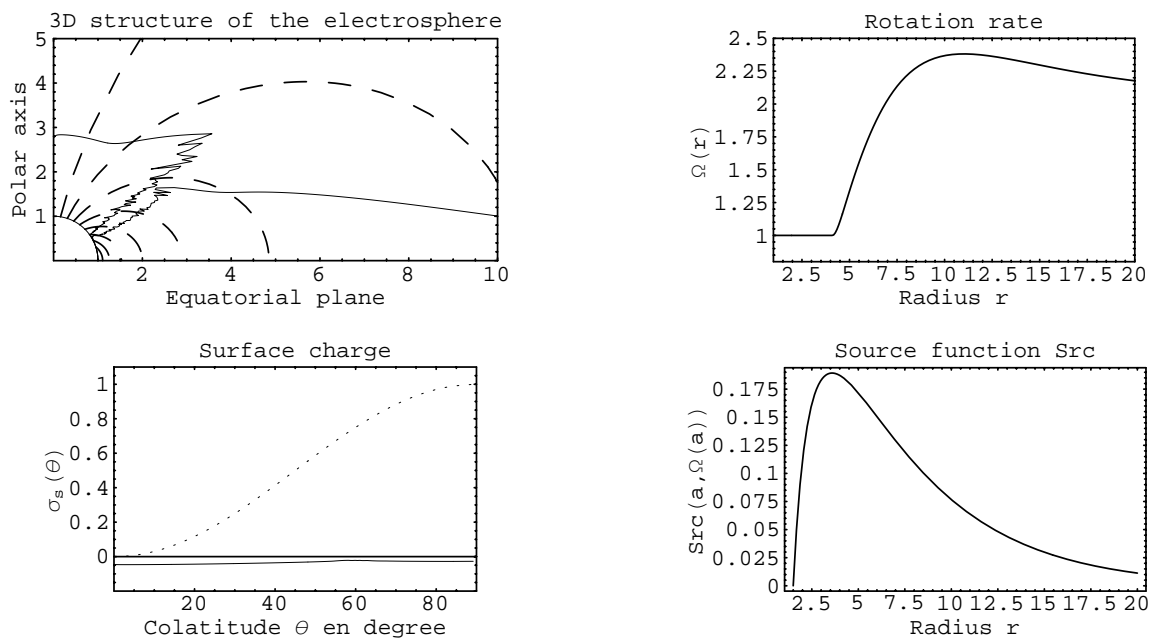

Fig. 14. Change in the electrospheric structure for the model $Q=3 Q_{\mathrm{c}}$ when a source of electron-positron pairs is added in the vacuum gaps. The quantities represented are as in Fig. 2. If the stellar radius is conveniently increased, we retrieve the equilibrium situation described in Sect. 4, in which the gaps were assumed to be stable against pair formation. A non negligible negative surface charge remains present over the star's surface because the presence of pair creation breaks full self-consistency.

no distinction between the star and the corotating electrosphere. The parameter $R_{\mathrm{p}}$ is but a scale factor which defines the radius of the electrically equivalent star.

This remark is illustrated in Fig. 14. Starting with the model with a total charge $Q=3 Q_{\text {c }}$ shown in Fig. 3 , we have added an arbitrary source of $\mathrm{e}^{-} \mathrm{e}^{+}$defined in the interval $a \in\left[0, \frac{1}{3}\right]$ by

$\operatorname{Src}^{ \pm}(a, \Omega(a))= \pm 2 \exp (-0.1 / a)\left(\frac{1}{3}-a\right)\left|\Omega(a)-\Omega_{*}\right|$

This source term has been chosen such that there be no pair creation very near the neutron star surface (factor $1 / 3-a$ ) nor very far away from it (factor $\exp (-0.1 / a))$, nor when there is corotation with the star (factor $\left|\Omega(a)-\Omega_{*}\right|$ ), because such magnetic surfaces are in this case entirely electrically connected to the star surface, and the gaps vanish on them. Although the geometry is slightly altered, it does not differ significantly from the previous results where the gaps had been assumed to be stable against pair formation, except for accounting for a change of the normalization radius of the corotating sphere $R_{*}$. This simple change of scale brings us back to the situation described in Sect. 4.

Negative surface charge remains on the crust. The algorithm in fact could not converge. This is because the equilibrium state of Fig. 14 is not self-consistently determined due to the presence of pair sources. If the pair cascade were to be stopped, charge would move back to the electrosphere to annihilate positive particles located in the disc restauring the situation described in Sect. 4. To maintain some marginal activity in the vacuum gaps, it would be necessary to allow particles to somehow escape from the system (via the equatorial plane and the poles) setting up an electric circuit. Describing this regime is the subject of our next papers.

\section{Conclusion}

In this paper the existence and viability of self-consistent pulsar electrospheres, finite in extent and in electrostatic equilibrium, have been proved in the case of aligned rotators. Large vacuum gaps separate the corotating polar regions from a differentially rotating belt. Compared with similar previous investigations by Krause-Polstorff \& Michel (1985b), we obtain more extended and much better results, overcoming their problem of an empty cone about the polar axis due to the artifact of the quantization of the surface charge. Moreover the shape of the electrosphere is in our case well defined, allowing us to obtain smooth rotation curves without any more noise than allowed by roundoff errors. We have also investigated the effect of charge loss from the polar caps and we have shown that it self-saturates in the absence of return current precisely by developing a charged electrostatic dome above them, as pictured by the entirely electrostatic model. Therefore the system is unlikely to evolve to a state where it emits a wind with no net charge loss.

Contrary to what is generally believed, the vacuum breakdown of these large gaps by $\mathrm{e}^{ \pm}$pair creation is not efficient enough to cause the filling-up of the whole magnetosphere up to the light surface by leptonic plasma. This statement unambiguously holds true for pulsars with periods in the range of a second. For the class of millisecond pulsars the conclusion remains unclear and would require a more precise evaluation of pair-creation effects as well as a relativistic description of the electrosphere.

There is however more to the structure of such electrospheres than just their axisymmetric equilibrium properties. The existence of a differentially rotating disk opens up the question of the stability of the plasma in such a configuration. This will be the purpose of a future paper where this question will be studied. It will be shown that the plasma is indeed most often unstable to the so called 
diocotron instability and the consequences of this situation will be studied.

\section{Appendix A: Non existence of star/vacuum contact}

In this appendix we show that no vacuum gap can exist between the polar regions of the electrosphere and the neutron star when electrostatic equilibrium is achieved. Such a gap would be bordered on one side by the star's surface and on the other side by another force free surface $S$ where the electric and magnetic fields should also be perpendicular to each other. The electric potential at these surfaces should then satisfy the condition $e_{\mathrm{B}} \cdot \nabla \phi=0$, where $\boldsymbol{e}_{\mathrm{B}}$ is the unit vector in the direction of the magnetic field. As will be seen the proof more generally shows that when electrostatic equilibrium is achieved the star must be surrounded by corotating electrospheric charge at any latitude.

At the stellar surface the potential equals the corotation one and in the nearby vacuum, if any, it satisfies Laplace equation, the general solution of which can be expressed as

$\phi=\sum_{l=0}^{+\infty}\left(a_{l} R^{l}+\frac{b_{l}}{R^{l+1}}\right) P_{l}(\cos \theta)$

its gradient being:

$$
\begin{aligned}
\nabla \phi= & \sum_{l=0}^{+\infty}\left(l a_{l} R^{l-1}-\frac{(l+1) b_{l}}{R^{l+2}}\right) P_{l}(\cos \theta) \boldsymbol{e}_{\mathrm{R}} \\
& +\frac{1}{R} \sum_{l=0}^{+\infty}\left(l a_{l} R^{l}+\frac{b_{l}}{R^{l+1}}\right) \frac{\mathrm{d}}{\mathrm{d} \theta}\left(P_{l}(\cos \theta)\right) \boldsymbol{e}_{\theta} .
\end{aligned}
$$

The potential $\phi$ must reduce to the corotation potential

$$
\phi_{\mathrm{c}}(R, \theta)=\frac{Q_{\mathrm{tot}}-Q_{\mathrm{el}}-Q_{\mathrm{imel}}}{4 \pi \varepsilon_{0} R_{*}}-\frac{1}{3} \Omega_{*} B_{*} R_{*}^{2} P_{2}(\cos \theta)(
$$

at the stellar surface. If, at electrostatic equilibrium, any part of the stellar surface is to be in contact with vacuum we should also enforce the condition that this part of the stellar surface be force free, i.e. that $\boldsymbol{e}_{\mathrm{B}} \cdot \boldsymbol{\nabla} \phi=0$ at $R=R_{*}$. This allows to identify term by term the coefficients $a_{l}$ and $b_{l}$ so that the potential in the vacuum region which is connex with this part of the star's surface can be expressed as:

$$
\begin{aligned}
\phi(R, \theta)= & \frac{Q_{\mathrm{tot}}-Q_{\mathrm{el}}-Q_{\mathrm{imel}}-Q_{\mathrm{c}}}{4 \pi \varepsilon_{0} R_{*}}+\frac{Q_{\mathrm{c}}}{4 \pi \varepsilon_{0} R} \\
& -\frac{1}{5}\left[\frac{2}{3} \Omega_{*} B_{*} R^{2}+\frac{\Omega_{*} B_{*} R_{*}^{5}}{R^{3}}\right] P_{2}(\cos \theta) .
\end{aligned}
$$

It is already noted that this solution contains a term which is growing with $R$, which would conflict with boundary conditions at infinity if that region were to extend that far. This then shows that the vacuum region which is connex to the star cannot reach to infinity, but, if it exists, it must come in the form of vacuum bubbles of limited extent included in charged electrospheric material. The corresponding electric field in such a vacuum region, $\boldsymbol{E}=-\boldsymbol{\nabla} \phi$, is given by

$$
\begin{aligned}
\boldsymbol{E}(R, \theta)= & \frac{Q_{\mathrm{c}}}{4 \pi \varepsilon_{0} R^{2}} \boldsymbol{e}_{\mathrm{R}}+\frac{1}{5}\left[\frac{4}{3} \Omega_{*} B_{*} R-3 \frac{\Omega_{*} B_{*} R_{*}^{5}}{R^{4}}\right] \\
& \times P_{2}(\cos \theta) \boldsymbol{e}_{\mathrm{R}}-\frac{3}{5} \sin \theta \cos \theta \\
& \times\left[\frac{2}{3} \Omega_{*} B_{*} R+\frac{\Omega_{*} B_{*} R_{*}^{5}}{R^{4}}\right] \boldsymbol{e}_{\theta}
\end{aligned}
$$

The solution in any vacuum region connex to the star being now determined, it becomes possible to examine whether $\boldsymbol{e}_{\mathrm{B}} \cdot \boldsymbol{\nabla} \phi=0$ can also vanish on another surface than the star's crust and so to determine the outer boundary of any such bubble. The projection of the electric field on the magnetic line is calculated to be equal to:

$$
\begin{gathered}
\boldsymbol{e}_{\mathrm{B}} \cdot \boldsymbol{E}=-\frac{3}{5} \sin ^{2} \theta \cos \theta\left[\frac{2}{3} \Omega_{*} B_{*} R+\frac{\Omega_{*} B_{*} R_{*}^{5}}{R^{4}}\right]+2 \cos \theta \\
\times\left[\frac{Q_{\mathrm{c}}}{4 \pi \varepsilon_{0} R^{2}}+\frac{\Omega_{*} B_{*}}{5}\left(\frac{4}{3} R-3 \frac{R_{*}^{5}}{R^{4}}\right) P_{2}(\cos \theta)\right] \cdot \quad(\text { A. } 6)
\end{gathered}
$$

Could it vanish, for non-zero $\Omega_{*}$, on a surface $S$ other than the stellar surface? The trivial solution $\cos \theta=0$ is eliminated because it corresponds to the equatorial plane. Since we already imposed the stellar surface to be force free the quantity $\left(R-R_{*}\right)$ can be factored out in the r.h.s. of Eq. (A.6) and, after simplification, the search for the outer boundary $S$ boils down to solving the following equation of degree four for the reduced radial distance $x=R / R_{*}$ of the point on this surface with colatitude $\theta$ :

$$
\begin{aligned}
\left(\frac{2}{3}-\frac{6}{5} \cos ^{2} \theta\right) x^{4} & +\left(\frac{2}{3}-\frac{6}{5} \cos ^{2} \theta\right) x^{3}+\left(\frac{2}{3}-\frac{6}{5} \cos ^{2} \theta\right) x^{2} \\
& -\frac{6}{5} x \cos ^{2} \theta-\frac{6}{5} \cos ^{2} \theta=0 .
\end{aligned}
$$

Note that this equation does not contain the total charge of the system $Q$ but only the central charge $Q_{\mathrm{c}}$ and therefore the proof below is valid for any value of $Q$. We first consider the exceptional case when $\left(\frac{2}{3}-\frac{6}{5} \cos ^{2} \theta\right)=0$ and Eq. (A.7) reduces to $x+1=0$. The latter has no strictly positive root, which means that the surface $S$, if it exists, has no point at the colatitude such that $\cos ^{2} \theta=5 / 9$. In the general case when $\left(\frac{2}{3}-\frac{6}{5} \cos ^{2} \theta\right) \neq 0$, Eq. (A.7) can be rewritten in the form

$x^{4}+x^{3}+x^{2}=\frac{9 \cos ^{2} \theta}{5-9 \cos ^{2} \theta}(x+1)$.

A physically valid solution would correspond to $x \geq 1$. When $\cos ^{2} \theta \geq 5 / 9$, the right hand side of Eq. (A.8) is negative for $x>0$ an no such solution can be found, which means that the outer edge of any vacuum bubble connex to the star cannot be found in this circumpolar angular sector. When $\cos ^{2} \theta$ decreases just below $5 / 9$ the function of $\theta$ on the r.h.s. of Eq. (A.8) becomes infinitely large and positive and a solution larger than unity to the Eq. (A.8) can be found. With decreasing $\cos \theta$, however this function 
decreases and for $\cos ^{2} \theta \leq 1 / 3$, no solution larger than unity exists for $x$ anymore. This means that any vacuum bubble between star and electrosphere must be confined to the angular sector $1 / 3 \leq \cos ^{2} \theta \leq 5 / 9$. However, only for one value of $\cos ^{2} \theta$, namely for $\cos ^{2} \theta=1 / 3$ can the outer edge of such an hypothetic bubble join the star's surface, whereas two such contacts would be needed to construct a consistent solution since we have shown that the bubble cannot open up to infinity. This altogether means that contact between the star's surface and vacuum must be restricted to the single point $\cos ^{2} \theta=1 / 3$, where the Goldreich Julian charge density vanishes. Our solutions indeed exhibit this property.

\section{Appendix B: Uniqueness of electrospheric solutions}

In this appendix we show that an electrospheric solution in full electrostatic equilibrium is unique for a given total charge $Q_{\text {tot }}$ of the system and for a given star's rotation rate $\Omega_{*}$. Consider two supposedly distinct solutions for which the potential are $\phi_{1}(\boldsymbol{r})$ and $\phi_{2}(\boldsymbol{r})$ resp. and the associated charge density $\rho_{1}(\boldsymbol{r})$ and $\rho_{2}(\boldsymbol{r})$. The difference $\psi(\boldsymbol{r})=\phi_{2}(\boldsymbol{r})-\phi_{1}(\boldsymbol{r})$ is a solution of Poisson's equation in all space, with source $D(\boldsymbol{r})=\rho_{2}(\boldsymbol{r})-\rho_{1}(\boldsymbol{r})$, subject to the boundary condition that $\psi(\boldsymbol{r})$ approaches zero at infinity, as $\phi_{1}(\boldsymbol{r})$ and $\phi_{2}(\boldsymbol{r})$ required to do. The source has however some special properties. Indeed, the charge density $\rho_{1}(\boldsymbol{r})$ and $\rho_{2}(\boldsymbol{r})$ are identical inside the star and in the common part of the corotating regions of the two electrospheres, being equal to the Goldreich-Julian charge density associated to $\Omega_{*}$. Therefore $D(\boldsymbol{r})$ vanishes in the star and in these corotating regions as it does wherever no electrospheric charge is present in either of the two solutions. The regions of non-zero source term $D(\boldsymbol{r})$ are to be found in the polar region, in the volume which is not common to the two domes, and in the equatorial corotating belt, to the volume which pertains one corotating region but not the other, and in the volume formed by the union of the two differentially rotating disks. The potentials $\phi_{1}(\boldsymbol{r})$ and $\phi_{2}(\boldsymbol{r})$ are, up to an additive constant (see Eq. (8)), both equal to the corotation potential in the intersection of the corotating regions of the two electrospheres. Hence, the difference $\psi(\boldsymbol{r})$ is a constant in this region which encompasses in particular the volume of the star itself. Since $\psi(\boldsymbol{r})$ is a solution of Poisson equation with source term $D(\boldsymbol{r})$, we may question whether such a local constancy of $\psi(\boldsymbol{r})$ is consistent with a non-zero difference $D(\boldsymbol{r})$. The answer is definitely no. This can be seen as follows: the potential created by the charge distribution $D(\boldsymbol{r})$ is

$\psi(\boldsymbol{r})=\frac{1}{4 \pi \varepsilon_{0}} \iiint \frac{D\left(\boldsymbol{r}^{\prime}\right)}{\left\|\boldsymbol{r}-\boldsymbol{r}^{\prime}\right\|} \mathrm{d}^{3} \boldsymbol{r}^{\prime}$

and the Green's function $1 /\left\|\boldsymbol{r}-\boldsymbol{r}^{\prime}\right\|$ can be expanded in Legendre polynomials (see Jackson 1975) as

$\frac{1}{\left\|\boldsymbol{r}-\boldsymbol{r}^{\prime}\right\|}=\sum_{l=0}^{+\infty} \frac{r_{<}^{l}}{r_{>}^{l+1}} P_{l}(\cos \gamma)$ where $\gamma$ is the angle between $\boldsymbol{r}$ and $\boldsymbol{r}^{\prime}$ and we have introduced the notation $r_{<}=\min \left(r, r^{\prime}\right)$ and $r_{>}=\max \left(r, r^{\prime}\right)$. Using the equality

$$
\begin{aligned}
& P_{l}(\cos \gamma)=P_{l}(\cos \theta) P_{l}\left(\cos \theta^{\prime}\right) \\
& \quad+2 \sum_{m=1}^{l} \frac{(l-m) !}{(l+m) !} P_{l}^{m}\left(\cos \theta^{\prime}\right) \cos \left(m\left(\varphi-\varphi^{\prime}\right)\right)
\end{aligned}
$$

and performing the integration, the term in $\cos \left(m\left(\varphi-\varphi^{\prime}\right)\right)$ vanishes and the potential inside the star is then

$$
\begin{aligned}
& \psi(\boldsymbol{r})=\frac{1}{4 \pi \varepsilon_{0}} \\
& \times \sum_{l=0}^{+\infty} \iiint \frac{r^{l}}{r^{\prime l+1}} D\left(\boldsymbol{r}^{\prime}\right) P_{l}(\cos \theta) P_{l}\left(\cos \theta^{\prime}\right) \mathrm{d}^{3} \boldsymbol{r}^{\prime}
\end{aligned}
$$

which is of the form

$\psi(\boldsymbol{r})=\sum_{l=0}^{+\infty} A_{l} r^{l} P_{l}(\cos \theta)$

where the coefficients $A_{l}$ are determined by

$A_{l}=\frac{1}{4 \pi \varepsilon_{0}} \iiint \frac{D\left(\boldsymbol{r}^{\prime}\right)}{r^{\prime l+1}} P_{l}\left(\cos \theta^{\prime}\right) \mathrm{d}^{3} \boldsymbol{r}^{\prime}$

Now, for $\psi(\boldsymbol{r})$ to be a constant in the volume of the star, it is necessary that only $A_{0}$ be non-vanishing. The other integrals should all vanish, which can be seen to imply thanks to an inversion transformation in $r^{\prime}$ that the distribution $D(\boldsymbol{r})$ should have no multipolar moment of any order but for the monopolar one. This charge distribution should then be spherically symmetric. But this is not possible in the present case because the existence of vacuum gaps implies that $D(\boldsymbol{r})$ vanishes on the sphere of radius $r^{\prime}$ wherever this sphere intersects the region common to the vacuum gaps of both solutions. Since these gaps always contain the null Goldreich-Julian density, such a region necessarily exists on any sphere. Spherical symmetry would then imply that $D(\boldsymbol{r})$ in fact vanishes over all the surface of such a sphere, and since this should be so for any sphere, it eventually implies that $D(\boldsymbol{r})$ vanishes everywhere. This establishes the uniqueness of the charge distribution $\rho(\boldsymbol{r})$ of the electrospheric solution for given $Q_{\text {tot }}$ and $\Omega_{*}$.

\section{References}

Abramowitz, M., \& Stegun, I. A. 1965, Handbook of mathematical functions (Dover, NY.)

Appl, S., \& Camenzind, M. 1993, A\&A, 270, 71

Arons, J. 1991, IAU coll. 128: The Magnetospheric Structure and Emission Mechanisms of Radio Pulsars' (Logow, Poland)

Asseo, E., Beaufils, D., \& Pellat, R. 1984, MNRAS, 209, 285

Beskin, V. S., Gurevich, A. V., \& Istomin, Ya. N. 1983, ZhETF, 85,401

Beskin, V. S., Gurevich, A. V., \& Istomin, Ya. N. 1993, Physics of the pulsar magnetosphere (Cambridge Univ Press.)

Bogovalov, S. V. 2001, A\&A, 371, 1155 
Cheng, A., Ho, C., \& Ruderman, M. 1986, ApJ, 300, 500

Cheng, A., Ruderman, M., \& Sutherland, P. 1976, ApJ, 203, 209

Contopoulos, I., Kazanas, D., \& Fendt, C. 1999, ApJ, 511, 351

Erber, T. 1966, Rev. Mod. Phys., 38, 626

Flowers, E. G., Ruderman, M. A., Lee, J.-F., et al. 1977, ApJ, 215,291

Goldreich, P., \& Julian, W. H. 1969, ApJ, 157, 869

Goldreich, P., \& Julian, W. H. 1970, ApJ, 160, 971

Hirotani, K. 2000, MNRAS, 317, 225

Hofmann, B., Herold, H., \& Ruder, H. 1996, PASPC, 105, 429

Holloway, N. J. 1973, Nature Phys. Sc., 246, 6

Holloway, N. J., \& Pryce, M. H. L. 1981, MNRAS, 194, 95

Jackson, E. A. 1976, ApJ, 206, 831

Jackson, E. A. 1979, ApJ, 227, 266

Jackson, J. D. 1975, Classical Electrodynamics, 2nd edition (John Wiley and Sons)

Jones, P. B. 1986, MNRAS, 218, 477

Krause-Polstorff, J., \& Michel, C. F. 1985, A\&A, 144, 72

Krause-Polstorff, J., \& Michel, C. F. 1985, MNRAS, 213, 43

Li, Z. Y., Chiueh, T., \& Begelman, M. 1992, ApJ, 394, 459

Mestel, L. 1994, Space Sci. Rev., 68, 337

Michel, F. C. 1973, ApJ, 180, 207
Michel, F. C. 1974, ApJ, 192, 713

Michel, F. C. 1979, ApJ, 227, 579

Michel, F. C. 1991, Theory of neutron star magnetospheres (the University of Chigago press)

Mueller, E. 1984, A\&A, 130, 415

Muslimov, A., \& Harding, A. 1997, ApJ, 485, 735

Muslimov, A., \& Tsygan, A. 1992, MNRAS, 255, 61

Neuhauser, T., Koonin, S., \& Langanke, K. 1987, Phys. Rev. A, 36,4163

Neukirch, T. 1993, A\&A, 274, 319

Pilipp, W. G. 1974, ApJ, 190, 391

Rezzolla, L., Ahmedov, B., \& Miller, J. 2001, MNRAS, 322, 723

Ruderman, M. A., \& Sutherland, P. G. 1975, ApJ, 196, 51

Rylov, Yu. A. 1976, SvA, 20, 23

Rylov, Yu. A. 1977, Ap\&SS, 51, 59

Rylov, Yu. A. 1985, Ap\&SS, 117, 5

Shibata, S. 1989a, Ap\&SS, 161, 145

Shibata, S. 1989b, Ap\&SS, 161, 187

Smith, I. A., Michel, F. C., \& Thacker, P. D. 2001, MNRAS, 322, 209

Thacker, P. D., Michel, F. C., \& Smith, I. A. 1998, Rev. Mex. Astron. Astrofís., 7, 211 